\title{
Discovery of Chalcone-Based Hybrid Structures as High Affinity and Site-Specific Inhibitors Against COVID-19; a Study Based on Various Host-Based and Viral Targets
}

\section{Mehdi Valipour}

Mazandaran University of Medical Sciences

Hamid Irannejad ( $\square$ irannejadhamid@gmail.com )

Mazandaran University of Medical Sciences

\section{Research Article}

Keywords: (SARS-CoV-2), COVID-19, HBATs, Severe Acute Respiratory Syndrome Coronavirus 2

Posted Date: September 16th, 2021

DOI: https://doi.org/10.21203/rs.3.rs-877666/v1

License: (c) (1) This work is licensed under a Creative Commons Attribution 4.0 International License. Read Full License 


\section{Abstract}

3-Chymotrypsin-like protease (3CLpro) and papain-like protease (PLpro) of Severe Acute Respiratory Syndrome Coronavirus 2 (SARS-CoV-2) are at the forefront of attention for finding therapeutic agents for the treatment of COVID-19. In addition, proper modulation of host-based antiviral targets (HBATs) has also been highlighted by scientists as a promising approach for the suppression of the SARS-CoV-2. Previous studies indicated that some natural-based chalcones have significant inhibitory effect on the coronavirus enzymes 3CLpro and PLpro as well as modulation of some HBATs. In this study, a computational screening was performed to investigate the affinity of our compound library consisting of 757 chalcone-based structures (CHA-1 to CHA-757) for inhibiting the 3CLpro and PLpro enzymes, and also against twelve selected host-based targets. The twelve selected HBATs were chosen based on their involvement in viral reproduction. Our results indicated that CHA-12 (VUF 4819) is the most potent and a multi-target inhibitor in our chemical library over all viral and host-based targets. Correspondingly, CHA-

384 and its congeners containing ureide moieties were found to be potent and selective 3CLpro inhibitors, and benzotriazole moiety in CHA-37 was found to be a main fragment for inhibiting the 3CLpro and PLpro. Surprisingly, our results were fully consistent with recent reports on the site-specific 3CLpro inhibitors. The results also indicate that the ureide and sulfonamide moieties are integral fragments for the optimum 3CLpro inhibition while occupying the S1 and S3 subsites. Finding the multi-target inhibitor CHA-12, previously reported as a LTD4 antagonist for the treatment of inflammatory pulmonary diseases, prompted us to suggest it as a concomitant antiviral agent for relieving respiratory symptoms and suppressing COVID-19 infection.

\section{Introduction}

Coronaviruses usually cause mild to severe respiratory infections. Some of the new strains of these viruses, such as Severe Acute Respiratory Syndrome (SARS-CoV) and Middle East Respiratory Syndrome (MERS-CoV), have caused terrible epidemics in the last two decades. In December 2019 in Wuhan, China, a new strain of the coronavirus called Severe Acute Respiratory Syndrome Coronavirus 2 (SARS-CoV-2) triggered a unique pandemic called COVID-19, which had an unprecedented rate of transmission and infected all over the world. Examination of the genome sequence of this new emerging virus showed that its genome is very similar to SARS-CoV (approximately 80\%) ${ }^{1}$. Therefore, results of previous studies on the treatment of SARS-CoV as one of the most accessible sources for finding a cure for SARS-CoV-2 were again considered and used by scientists. Today, many scientists are finding ways to treat the COVID-19. As a result of these efforts, various compounds have been identified which have notable inhibitory effects against SARS-CoV-2. Previously, numerous studies have reported notable antiviral activities for compounds derived from natural sources ${ }^{2,3}$. Some of these compounds act through direct damage to virus components, while others disrupt vital virus functions such as entering the host cell, replication, or other host-virus interactions ${ }^{4,5}$.

Treatment of COVID-19 requires careful consideration of cellular and molecular mechanisms to accurately identify the components as well as pathogenic mechanisms of the SARS-CoV-2. Structural 
and functional studies have shown that the virus is composed of various structural (SPs) and nonstructural proteins (NSPs) ${ }^{6}$. The virus has four major glycoproteins in its structure: spike protein (S), membrane protein $(M)$, envelope protein (E), and nucleocapsid $(N)$ protein. SARS-CoV-2 spike $(S)$ glycoproteins attach to appropriate host cells and cause virus entry, while proteins $M, E$, and $N$ are more involved in the assembly, replication, release of the virus particles, and physical protection. In addition to SPs, 15 NSPs have been identified for the SARS-CoV-2 so far ${ }^{7}$. Depending on the importance of their role in the survival and replication of the virus, each of them can be considered as a potential target. 3Chymotrypsin-like protease (3CLpro) and papain-like protease (PLpro) enzymes are the most important NSPs that play a vital role in the processing of SARS-CoV-2 polyproteins and the production of the other NSPs. These proteases are being considered by scientists as important targets for the design of SARSCoV-2 inhibitors ${ }^{8-10}$.

Recently, the active site of the SARS-CoV-2 3CLpro was well characterized and realized that the active site has four subsites named S1, S1', S2, and S3. The 3CLpro is a cysteine protease, and His41 and Cys145 comprise the catalytic dyad. Dimerization of the enzyme completes the active site and forms the oxyanion hole for substrate specificity. The $\mathrm{S} 1$ subsite has two key residues His 163 , which is interacting with the pyridine nitrogen or with nitrogen-containing heterocycles, and Glu166 which is a key amino acid in the formation of hydrogen bonds to the amide or ureide substructures of the inhibitors. The S2 subsite of SARS-CoV-2 has a small and hydrophobic space termed as "aromatic wheel" mainly consisting of Met49 and Met165. The S3 subsite has been revealed to be explored and occupied by the sulfonamide group of the inhibitors and interacts with hydrogen bonding to Gln189. This space is exposed to the solvent and fragments located in this space can be oriented to the solvent. This space consists of His164, Met165, and Asp187. These subsites are mainly used for the design of novel inhibitors simultaneously occupying two or three subsites for higher affinity by fragment merging or growing ${ }^{11}$.

Although many direct-acting antiviral drugs (DAADs) are designed to inhibit virus components, overuse of these agents can eventually lead to undesirable effects such as drug resistance ${ }^{12,13}$. Viruses broadly use host cell capabilities known as host-based antiviral targets (HBATs) to replicate their own particles. Therefore, targeted regulation of these features can help to inhibit virus pathogenicity. Because HBATs are not genetically controlled by the virus genome, it is expected that inhibiting viruses through regulating these targets does not lead to the drug resistance ${ }^{14}$.

Almost, two years after the emerge of the SARS-CoV-2, management of HBATs for the treatment of COVID-19 has become of great interest for scientists. Due to the homology and high genetic similarity of SARS CoV-2 to SARS-CoV ( 80\% similarity) and MERS-CoV ( 50\% similarity), it is well established that these viruses use a similar mechanism to enter the host cells, and infect their host by binding to the angiotensin-converting enzyme II (ACE2) receptors on the surface of the host cell ${ }^{15,16}$. Disrupting host cell entry, which is the first step in the viral life cycle, can be a sensible treatment and preventive strategy. Role of the host cathepsin cysteine protease enzyme is crucial for infecting ACE2 receptor-containing cells and the entry of the SARS-CoV-2 through endocytosis. So, cathepsin L could be considered as a high 
potential HBAT for therapeutic interventions ${ }^{17}$. The p38 Mitogen-Activated Protein Kinase (p38 MAPK) is another highlighted HBAT for the treatment of COVID-19. Since this signaling pathway plays a critical role in host-inflammatory responses and release of cytokines, and is also regulated by coronaviruses for infection, accordingly, this pathway was introduced by scientists as a promising target for the treatment of COVID-19 ${ }^{18}$. Cyclin-dependent kinases (CDK) are another highlighted HBATs for the treatment of COVID-19 and play a prominent role in the cell proliferation. Basically, viruses alter the functioning of CDKs to favor viral replication and hence, some recent studies considered the development of the CDK inhibitors to be an important strategy for achieving broad-spectrum antivirals that can be used to treat COVID-19 ${ }^{19}$. Some scientists have recently proposed dihydrooroate dehydrogenase (DHODH) as an attractive HBAT for the development of broad-spectrum antivirals, which provides more treatment options for COVID-19 through its dual mechanism of antiviral action and immune system regulation 20 .

Chalcones are a special structural class of natural compounds that can potentially inhibit SARS-CoV-2. In previous investigations, some chalcones have been introduced which showed significant potential to inhibit SARS-CoV 3CLpro and PLpro enzymes. In a study by Ji-Young Park et al., it was found that some natural-based chalcones Xanthoangelol E, Xanthoangelol D, Xanthoangelol B, and Xanthoangelol $F$ (Fig. 1) exert anti-SARS-CoV activity by inhibiting the 3CLpro and PLpro enzymes through competitive (for 3CLpro) and non-competitive (for PLpro) inhibitory mechanisms ${ }^{21}$. Some other studies have also reported that chalcone-based structures have important effects in inhibiting these enzymes ${ }^{22-24}$. In addition, some studies have reported that chalcones can disrupt vital processes such as virus replication by modulating HBATs ${ }^{25}$. Given that many synthetic or natural derivatives of chalcones have been identified so far, it makes sense to search for finding new and active derivatives of chalcone-based structures against SARS-CoV-2.

In this study, we have tried to find and identify active chalcones against the SARS-CoV-2 PLpro and 3CLpro enzymes within a compound library of 757 chalcone-based structures using computational methods, and also to evaluate structural relationships between these compounds and the important previously reported inhibitors. In addition, the affinity of these compounds against a wide range of HBATs was evaluated in silico to identify hit compounds with the highest potential therapeutic activity. Subsequently, a part of the aim in this study was to discover and propose subsite-specific inhibitors of the 3CLpro and PLpro targets.

In the current study, a wide range of important HBATs (12 targets) which were previously reported as potential targets for the treatment of viral diseases including COVID-19 were selected. These targets are included as cathepsin L, various types of CDKs, p38 MAPK, histone deacetylase 2 (HDAC2), dihydroorotate dehydrogenase (DHODH), bromodomain-containing proteins (BRDs), and sigma-1 receptor. Table 1 lists the specific HBATs and their PDB code considered in this study along with the chemical structure of their co-crystal ligands and also cites credible articles that highlight the importance of these targets as key HBATs for the treatment of viral diseases such as COVID-19. 
Accordingly, a compound library consisted of 757 chalcone-based structures was screened virtually by molecular docking over the 14 molecular targets reported in Table 1, and the best hits were ranked and selected based on their docking score. Ranking and selection were performed based on a criteria described later in this study and the best hits were selected for molecular dynamics and then $\mathrm{MM} / \mathrm{PB}(\mathrm{GB}) \mathrm{SA}$ analysis. Finally, a literature study has been done for the introduced ultimate leads and their experimentally reported biological activities discussed.

\section{Results}

\section{Virtual screening}

Chalcone-based structures were searched in the PubChem database and after applying Lipinski's rule of five, 757 chalcone derivatives were downloaded and used for virtual screening by AutoDock Vina over the 14 selected host and virus-based targets reperesented in Table 1. The molecular targets used are included as p38 MAPK, Cathepsin L, CDK1, CDK2/CyclinA, CDK9/cyclinT1, ERK2, HDAC2, DHODH, CK2 alpha, BRD2, BRD4, and sigma-1 receptor, and virus-based targets 3CLpro and PLpro. Standard inhibitors baicalein (a selective 3CLpro inhibitor and the co-crystal ligand of PDB code: 6M2N), GRL0617 (a selective PLpro inhibitor), and hirsutenone (a dual inhibitor of the 3CLpro and PLpro) were used in our computational protocol as reference compounds, and validation was performed through re-dock of the co-crystal ligands in each target.

Docking scores of our compound library over the host and virus-based molecular targets are reperesented in Fig. 2 while each compound is presented by a single sign and each target by a different color. As shown in the figure, on average, the chalcone-based compounds showed higher affinities toward the active site of the targets DHODH (5ZF7), CK2 alpha' (5M4U), sigma-1 receptor (6DKI), and CDK9/cyclinT1 (3BLR) respectively compared to other HBATs. Although the average docking scores obtained for 3CLpro and PLpro enzymes are not remarkable, but probably this is caused by the presence of a superficial active site of these enzymes which is not a deep cavity. Comparison of the identified most active chalcones toward the two viral targets with their well-known standard inhibitors shows that these chalcones have a significant potential for the inhibition of the SARS-CoV-2 3CLpro and PLpro enzymes. Structural evaluation and analysis of these compounds led us to interesting results, which will be discussed in the following.

In order to choose the best hits with the highest docking score, two selection criteria were applied. First, the structures that were ranked 1 to 5 in their interaction with the active site of each HBATs were identified (Table 2). Second, those compounds which were ranked 1 to 10 in their interaction with the active site of the 3CLpro and PLpro, were also identified and selected. The latter was done in order to focus more precisely in the viral targets, and structures which had better rank in the 3CLpro and PLpro active sites were considered separately regardless of the host-based targets (Table 2). Finally, compounds that generated higher ranking with both host and virus-based targets were selected and highlighted for further structural and computational evaluations. For example, CHA-37 (rank 3 in PLpro with PDB code of 7JN2) 
was priotized over CHA-282 (rank 2 in PLpro with PDB code of 7JN2) due to the higher ranking of CHA-37 over the host-based targets compared to CHA-282.

By filtering the compounds through the selection criteria, some structures were identified that could selectively create remarkable interactions in the active sites of the 3CLpro or PLpro targets. While, some compounds were found to simultaneously create strong interactions on both the SARS-CoV-2 targets. Among all 757 chalcone-based compounds in the library, some compounds such as CHA-15, CHA-236, CHA-383, CHA-384, CHA-392, CHA-397, and CHA-485 showed selective affinity in the 3CLPro active site. On the other hand, CHA-7, CHA-11, CHA-37, CHA-44, and CHA-177 showed selectivity to the PLPro active site, and CHA-12, CHA-37, CHA-297, and CHA-378 showed interesting potential for simultaneous inhibition of both the 3CLpro and PLpro targets. In our opinion, having the high potential for simultaneous inhibition of the HBATs along with the viral enzymes is an important criterion for the selection of ligands for subsequent evaluations. For example, CHA-86, CHA-118, CHA-233, and CHA-282, were not selected for further study due to their lack of potential to inhibit HBATs, despite their high potential for inhibiting 3CLPro and PLPro enzymes. Among all compounds in our library, CHA-12, CHA-37, CHA-297, CHA-378, and CHA-384 are interesting compounds that generated an excellent binding profile in the active sites of the most targets (Fig. 3). Structural analysis and binding free energy calculations of these ligands in the viral targets showed interesting structure-activity relationships which are consistent with the experimental results previously reported for SARS-CoV/SARS-CoV-2 inhibitors.

\section{Molecular dynamics \& MM/PB(GB)SA analysis}

The best hits found by docking virtual screening were chosen for further molecular dynamics and $\mathrm{MM} / \mathrm{PB}(\mathrm{GB}) \mathrm{SA}$ analysis. Baicalin as a co-crystal ligand and a standard inhibitor of the 3CLpro and GRL0617 as a well-known and standard inhibitor of the PLpro were considered in our study. Hirsutenone was also used in our computational and structural analysis as an experimentally reported 3CLpro and PLpro inhibitor (Fig. 3). Correspondingly, CHA-12, CHA-384, CHA-378, CHA-297, CHA-37, compounds A and $\mathbf{B}$ were studied in our molecular dynamics and binding free energy evaluations in the 3CLpro active site. Similarly, ligands CHA-12, CHA-37, and CHA-378 were used in our molecular dynamics and binding free energy calculations in the PLpro active site (Fig. 3).

An overlay of all the RMSD plots of the simulations with the 3CLpro and PLpro with respect to the Calpha were calculated and are shown in Figs. $4 \mathrm{~A}$ and $4 \mathrm{~B}$ respectively. RMSD plot is a useful index to evaluate the stability of the protein and the complex during the simulation time. Totally, the convergence of the RMSD curve around a smaller value indicates favorable and high stability of the complex.

As seen in Fig. 4A, the 3CLpro-baicalin has minimal variations and oscillations during the simulation time of $10 \mathrm{~ns}$ which is in the range of $0.10-0.21 \mathrm{~nm}$ with an average value of 0.14 indicating a very stable complex. In the case of 3CLpro-CHA-12, the RMSD plot is in the range of $0.10-0.28 \mathrm{~nm}$ and the mean value is 0.16 . It has only a small rise in the $2 \mathrm{~ns}(0.28 \mathrm{~nm})$ but the ongoing part is a plateau and the smooth portion of the curve indicates its appropriate stability. For CHA-384 equilibrated in the 3CLpro during the simulation time of $10 \mathrm{~ns}$, the RMSD plot shows a very stable complex with minor oscillations 
which is in the range of $0.10-0.20 \mathrm{~nm}$ and the mean value of 0.14 . The CHA-37 and CHA-297-3CLpro equilibration has also a stable complex during the simulation with a varied range of $0.10-0.24 \mathrm{~nm}$ and a mean value of 0.17 . Hirsutanone and CHA-378 have also a stable condition in the 3CLpro active site indicated by its RMSD curve which is in the range of $0.10-0.26 \mathrm{~nm}$ and a mean value of 0.16 . Correspondingly, compounds $\mathbf{A}$ and $\mathbf{B}$ have also formed a stable complex with $3 \mathrm{CLpro}$ as indicated by their RMSD plot and a mean value of 0.14 (range $0.10-0.18 \mathrm{~nm}$ ) for compounds $A$ and 0.19 (range of $0.10-0.31 \mathrm{~nm}$ ) for compound $\mathbf{B}$ have been obtained. In the latter case, a moderate increase from 0.15 to $0.31 \mathrm{~nm}$ is observed in the interval time $4-7 \mathrm{~ns}$.

The RMSD plots for ligands equilibrated in the PLpro (Fig. 4B) are also indicative of their stable complexes during the simulation time. In the case of the co-crystal ligand and GRL0617-PLpro complexes, the varied RMSD values are in the range of $0.10-0.27 \mathrm{~nm}$ with a mean value of 0.18 . Correspondingly, CHA-12 has a very stable complex with PLpro in $10 \mathrm{~ns}$ within a range of $0.10-0.23 \mathrm{~nm}$ and an average value of 0.15. The mean RMSD values for CHA-37-PLpro, CHA-378-PLpro and hirsutenone-PLpro complexes are $0.14(0.10-0.23 \mathrm{~nm}), 0.15(0.10-0.23 \mathrm{~nm})$, and $0.15(0.10-0.25 \mathrm{~nm})$ respectively, and accordingly, all are in a very stable equilibrated conditions in the whole simulation time.

An overlay of all the RMSF plots of the simulations with 3CLpro and PLpro with respect to the C-alpha were calculated and are shown in Figs. 4C and 4D respectively. The RMSF curve of the simulation shows flexibility and dynamics of the protein main chain corresponding to each amino acid residue. Totally, the minimal fluctuations over the entire amino acid sequence indicate a stable structure, although flexible regions of the protein such as loops and the two ends of the protein normally have greater values of fluctuations compared to the internal parts and structured alpha-helix or beta-sheets. Overlay of all RMSF plots of the 3CLpro and PLpro shows that the average value for all the complexes is around $0.10 \mathrm{~nm}$ and the stability of complexes are in good accordance with the RMSD values. As seen in Fig. 4C, the RMSF curves of all ligands in the 3CLpro have been superimposed well on each other with minimal differences and clearly confirm high integrity and normal conditions for all dynamic simulations. The same fact also exists for all ligands equilibrated in the PLpro as shown by their well superimposed RMSF curves (Fig. 4D).

All the RMSD and RMSF plots together confirmed the minor geometrical and structural changes during the simulation time and conservation of the initial crystal structure of the protein before and after the dynamic process.

The trajectories of MD simulations were used for the covariance analysis in order to evaluate the dynamic motions of the protein. A $918 * 918$ covariance matrix based on the carbon-alpha fluctuations was diagonalized to generate eigenvalues against the eigenvectors and the first 50 eigenvectors showed $\geq 99 \%$ of the variance of motions for each dynamic simulation. Accordingly, a 2D projection of the two first principal components PC1 and PC2 that represent the dominant conformational fluctuations for all the ligands studied in the $3 \mathrm{CL}$ pro and PLpro active sites are visualized in Figs. 4E and 4F respectively. The results obtained by covariance analysis are in good agreement with the RMSD and RMSF plots. 
Trace values of the covariance matrix before and after the diagonalizing are between 3.06 and 4.13 for all ligands studied in the 3CLpro except for compound $\mathbf{B}$ which is 6.05. In the latter case, the 2D projection of PC1 and PC2 of compound B is represented by orange circles in Fig. 4E which shows its more distribution over the wider space and clearly indicates its relatively lower stability of the protein compared to the others. This was also evidenced by its RMSD plot as discussed earlier.

The trace values of the covariance matrix before and after the diagonalizing for ligands studied in the PLpro are in the range of 3.69-5.48 (Fig. 4F).

In order to calculate the binding free energy of the selected hit compounds, the trajectories of the MD simulations were used for the Molecular Mechanics/Poisson Boltzmann (or Generalized Born) Surface Area $(M M / P B(G B) S A)$ analysis. Final results of the $M M / P B(G B) S A$ analysis as binding free energy $\left(\triangle G_{b}\right.$ in $\mathrm{kcal} / \mathrm{mol}$ ) are visualized in Figs. $5 A$ and $B$ respectively for the $3 C L$ pro and PLpro. Values of the energy components, total free energy $\left(\Delta \mathrm{G}_{\text {total }}\right)$, solvation energy $\left(\Delta \mathrm{G}_{\text {solv }}\right)$, gas- phase free energy $\left(\Delta \mathrm{G}_{\text {gas }}\right)$, and the interaction entropy (IE) for each compound studied in MD and MM/PB(GB)SA analysis along with their final binding free energy values $\left(\Delta G_{b}\right)$ are summarized in Tables 3 and 4 for the 3CLpro and PLpro respectively.

As shown in Fig. 5A, CHA-12 has the best binding affinity towards the 3CLpro among the compounds studied in our library with the binding free energy of -38.57 and $-32.36 \mathrm{kcal} / \mathrm{mol}$ respectively calculated by the GB and PB methods. Interestingly, CHA-12 has even more binding affinity than the 3CLpro cocrystal ligand baicalein $\left(\Delta \mathrm{G}_{\mathrm{b}}=-30.93\right.$ and $-26.40 \mathrm{kcal} / \mathrm{mol}$ calculated by the GB and PB respectively) and also better than the well-known 3CLpro inhibitor hirsutenone $\left(\Delta G_{b}=-24.12\right.$ and $-18.69 \mathrm{kcal} / \mathrm{mol}$ calculated by the GB and PB methods respectively) in the active site of the 3CLPro.

CHA-384 which is a hybrid of a chalcone and ureide moiety is in the second-order of affinity towards the 3CLPro $\left(\Delta \mathrm{G}_{\mathrm{b}}=-33.37\right.$ and $-25.13 \mathrm{kcal} / \mathrm{mol}$ respectively calculated by the $\mathrm{GB}$ and PB methods) and showed better binding energy than baicalein (in GB only) and hirsutenone. Based on the results, $\mathrm{CHA}-384$ was found and considered to be a selective 3CLpro inhibitor among the 757 chalcone-based compounds evaluated in this study.

Results of the GB calculations indicated that CHA-37 $\left(\Delta \mathrm{G}_{\mathrm{b}}=-31.09 \mathrm{kcal} / \mathrm{mol}\right.$ calculated by the $\mathrm{GB}$ method) is in the third order of the affinity towards the 3 CLpro and is more potent than baicalein, hirsutenone, and the sulfonamide-containing compounds CHA-297 and CHA-378. Results of the PB method also confirmed that CHA-37 $\left(\Delta \mathrm{G}_{\mathrm{b}}=-20.66 \mathrm{kcal} / \mathrm{mol}\right)$ has more binding affinity than hirsutenone, and the sulfonamide-containing compounds CHA-297 and CHA-378 towards the 3CLpro active site. However, the PB method indicated the higher affinity of baicalein $\left(\Delta G_{b}=-26.40 \mathrm{kcal} / \mathrm{mol}\right)$ over CHA-37 in the active site of the 3CLpro. The sulfonamide-containing compounds CHA-297 and CHA-378 have shown lower affinity than the standard inhibitors baicalein and hirsutenone and also less affinity than the CHA-12, CHA-384, and CHA-37 indicated by both the GB and PB methods. 
Fragment compound $A$ bearing sulfonamide moiety $\left(\Delta G_{b}=-11.96 \mathrm{kcal} / \mathrm{mol}\right)$ revealed less affinity than the ureide-containing compound $\mathbf{B}\left(\Delta \mathrm{G}_{\mathrm{b}}=-20.36 \mathrm{kcal} / \mathrm{mol}\right)$ by the $\mathrm{GB}$ method, but more potency than $\mathbf{B}$ calculated by the PB method (Table 3).

Energy components of binding clearly indicate that the high binding affinity of $\mathrm{CHA}-12$ reasoned by its favorable interaction entropy (IE) of -0.56 and its high enthalpic contribution represented by its $\Delta \mathrm{G}_{\text {gas }}$ of $-62.05 \mathrm{kcal} / \mathrm{mol}$ (Table 3). The diagram of the energy components shown in Figs. 6E and 6F clearly indicates that the van der Waals and electrostatic energies are more favored by CHA-12 than baicalein and CHA-384. Ortho-substitution of the phenyltetrazole ring by hydroxyl and ketone groups facilitates the formation of an intra-hydrogen bond between the oxygen atom of the ketone and proton of the hydroxyl group. This phenomenon restricts the free rotation of the chalcone linker and favors the entropy cost upon binding. Accordingly, the interaction entropy of CHA-12 is $-0.56 \mathrm{kcal} / \mathrm{mol}$ and hence much better than baicalein with a rigid structure $(\mathrm{IE}=0.84 \mathrm{kcal} / \mathrm{mol})$. The interaction entropy of ligands CHA-384 and hirsutenone are much more positive as represented in Table 3 and negatively impacts the binding energy. Diagrams of the energy components constituting the $\Delta \mathrm{G}_{\mathrm{T}}$ and $\Delta \mathrm{G}_{\mathrm{b}}$ of baicalein, CHA12, CHA-384, CHA-37 and hirsutenone in the active site of the 3CLpro are reperesented in Fig. 6.

In the case of the PLpro, CHA-12 was again found to be the most potent compound calculated by the $\mathrm{MM} / \mathrm{PB}(\mathrm{GB}) \mathrm{SA}$ method. CHA-12 with binding free energy $\left(\Delta \mathrm{G}_{\mathrm{b}}\right)$ of -32.86 and $-27.85 \mathrm{kcal} / \mathrm{mol}$ respectively calculated by the GB and PB methods was much better than the standard inhibitor GRL0617 $\left(\Delta G_{b}=-31.33\right.$ and $-23.65 \mathrm{kcal} / \mathrm{mol}$ respectively calculated by the $\mathrm{GB}$ and $\mathrm{PB}$ methods $)$ and hirsutenone $\left(\Delta \mathrm{G}_{\mathrm{b}}=-22.67\right.$ and $-16.52 \mathrm{kcal} / \mathrm{mol}$ respectively by the $\mathrm{GB}$ and $\left.\mathrm{PB}\right)$ in the active site of the PLpro.

Enthalpic contributions including van der Waals and electrostatic energies are the main factors favoring the total binding energy of CHA-12 as represented in Table 4 and Fig. 7. Also, the interaction entropy cost of CHA-12 is a small quantity of $+1.95 \mathrm{kcal} / \mathrm{mol}$. Therefore, $\mathrm{CHA}-12$ is considered a multi-functional compound affecting various virus and host-based biological pathways as discussed earlier in the virtual screening results of the docking scores of host-based targets.

Ligand $\mathbf{3 7}$ was shown to be a higher affinity PLpro inhibitor than the selective and standard PLpro inhibitor hirsutenone which is indicated by the PB results (CHA-37, $\Delta G_{b}=-19.20$; hirsutenone, $\Delta G_{b}=$ $-16.52 \mathrm{kcal} / \mathrm{mol}$ ), but the GB calculations afforded opposite results. CHA-37 has fewer van der Waals energies than CHA-12 as shown in Fig. 7 but with the same quantity of electrostatic energies. CHA-37 also has a very small entropy cost of $0.75 \mathrm{kcal} / \mathrm{mol}$.

The sulfonamide-containing compound CHA-378 was found to be a weak binder for the PLpro active site compared to CHA-12, CHA-37, and the standard inhibitors GRL0617 and hirsutenone. This weak binding affinity of CHA-378 was also observed for the 3CLpro as discussed earlier.

\section{Druglikeness, ADME, and toxicity}

Natural-based compounds quercetin and resveratrol are among the most important safe supplements suggested to combat COVID-19 as adjuvants ${ }^{94-96}$. Due to their valuable medicinal effects, these 
compounds have long been available in the market as oral supplements. Herein, the drug-likeness, ADME properties, oral bioavailability, and hepatotoxicity of the most important identified compounds CHA-12, CHA-37, CHA-378, and CHA-384 are compared to the quercetin and resveratrol as standard medicines. Based on the results of these evaluations, it is predicted that the most active compounds identified in this study have comparable or even better drug-likeness profiles compared to the standard compounds quercetin and resveratrol (Table 5).

\section{Oral bioavailability}

Oral bioavailability is often an important consideration for the discovery and development of bioactive molecules as therapeutic agents. Thus, understanding of the molecular properties that limits oral bioavailability is an important goal for drug researches. The most important parameters that are considered for evaluation of oral bioavailability include: lipophilicity, molecular size, molecular polarity, insolubility, insaturation of molecular structure, and molecular flexibility. As shown in Fig. 8, of the six parameters considered, CHA-12, CHA-37, CHA-384 and CHA-378 have five parameters in the desired range. Accordingly, these compounds are expected to have appropriate oral bioavailability.

\section{Cytochrome P450 interaction}

Hepatotoxicity is one of the most important issues in the process of discovering new drugs. The effects of the evaluated compounds on increasing or decreasing the function of cytochrome P450 enzymes have always been considered ${ }^{97}$. CYP3A4, CYP2D6, CYP2C9, and CYP2C19 isoforms are the most well-known subtypes of liver cytochrome P450 enzymes that play a vital role in drug metabolism and their effective or toxic concentrations in the body ${ }^{98}$. Because of the importance of this issue, we have tried to predict the effects of CHA-12, CHA-37, CHA-378, CHA-384, resveratrol, and quercetin on the liver enzymes, using computational methods. According to the results in Table 6, unlike standard drugs quercetin and resveratrol which inhibit CYP2C19 and CYP3A4, CHA-12, CHA-37 and CHA-384 are not expected to have inhibitory effects on these enzymes. Also, the effects of these ligands on the other cytochrome P450 enzymes (CYP2C9, CYP2D6 and CYP3A4) are similar to the effects of the standard drugs. Therefore, these ligands are predicted to have fewer hepatotoxic effects than the standard drugs.

\section{Discussion}

\section{CHA-12 as a multi-target inhibitor}

Leukotrienes are broncho-constrictors whose pharmacological effects mimic the pathological changes in asthma. The biological activity of leukotrienes is mediated by the activation of cysteine leukotrienes (CysLT1 and CysLT2), leading to bronchoconstriction, mucus secretion, and asthma-like conditions. Accordingly, CysLT1 (LTD4) receptor antagonists are considered as important therapeutic agents in the treatment of lung and respiratory diseases such as asthma. 
Results of the current study revealed that the hit compound $\mathrm{CHA}-12$ has a magic affinity towards all tested targets. Interestingly, CHA-12 was first introduced as a CysLT1 antagonist in 1997 in a study by Mariel E. Zwaagstra et al ${ }^{99}$. Among more than sixty compounds synthesized in that study, CHA-12 showed the best CysLT1 antagonistic activity in both in vitro and in vivo assays. In fact, patients infected with COVID-19 suffer from acute inflammation in the lung and respiratory system mostly due to the cytokine storm and having this important pharmacological activity of CHA-12 greatly increases the chance of CHA-12 to be a lead compound for treating COVID-19 complications in the respiratory system.

Impressive results of the virtual screening of the 757 chalcone-based compound library over 14 host and virus-based molecular targets and also the MM/PB(GB)SA analysis indicated that CHA-12 is a unique compound and has the highest affinity towards the 3CLpro and PLpro active sites. As shown in Fig. 9, CHA-12 fits well into the 3CLpro active site. Presence of the phenyltetrazole moiety in the structure of this compound structurally makes a partial similarity to losartan, an angiotensin II receptor blocker (ARB) reported to have remarkable effect for the treatment of COVID-19 (Fig. 10) ${ }^{100-104}$. In a recent HTS study performed by Zhengnan Shen et al, candesartan as an ARB was identified as one of a few compounds that showed inhibitory effects against SARS-CoV-2 PLpro, out of about 15,000 screened compounds ${ }^{105}$. The phenyltetrazole moiety of CHA-12 makes crucial interactions in the active site of the viral targets (especially PLpro) which are discussed later in the following.

The most important interactions of CHA-12 in the active site of 3CLpro are shown in Fig. 9. Totally, hydrogen bonds were found to be the main part of the binding affinity. Essentially, several hydrogen bondings were observed between the bound conformation of CHA-12 and the 3CLpro active site residues. In fact, two hydrogen bonds are formed by the interaction of His41 with the quinoline nitrogen and oxygen atom of the oxymethylene chain; one hydrogen bond exists between Glu166 and the $\mathrm{NH}$-tetrazole, another hydrogen bonding between Thr190 and phenolic hydroxyl group and finally, one hydrogen bonding also exists between Gln192 and the carbonyl oxygen atom. In addition, Pi-sulfur interactions of Met49 to the quinolone and phenyl rings and a Pi-sigma interaction of Thr25 with the pyridine ring of quinoline moiety and also a Pi-donor H-bond interaction of GIn189 with the phenyl ring of the chalcone backbone contribute to the stronger binding of CHA-12 in the 3CLpro active site.

Evaluation of the CHA-12 interactions at the PLpro active site shows that the tetrazole moiety forms two key hydrogen bonds with Lys157 and GIn269 residues, as well as a Pi-anion interaction with the Glu167. A hydrogen bonding also exists through phenolic hydroxyl group with Tyr264 and Tyr268 has three interactions of type Pi-Pi stacked, Pi-Pi T-shaped and amide-Pi stacked with the phenyl and quinoline moieties of CHA-12. There is also a Pi-alkyl interaction between Pro248 and the phenyl ring of $\mathbf{C H A - 1 2}$ (Fig. 9).

Therefore, reported relaxant effects of CHA-12 on pulmonary smooth muscles established by in vitro and in vivo assays ${ }^{99}$, along with its prominent predicted inhibitory effects on the all SARS-CoV-2 targets supports the discovery of a valuable compound for the treatment of COVID-19.

\section{Chalcones bearing sulfonamide moiety}


CHA-378 and CHA-297 having a sulfonamide moiety in their structure are also interesting compounds showed relatively acceptable results in the active site of the 3CLpro and PLpro targets. The CHA-378 generates strong interactions in the active sites of most HBATs including p38 MAPK (rank 10, docking score: $-9.0 \mathrm{kcal} / \mathrm{mol}$ ), Cathepsin L (rank 10, docking score: $-8.0 \mathrm{kcal} / \mathrm{mol}$ ), CDK2/CyclinA (rank 5, docking score: $-10.1 \mathrm{kcal} / \mathrm{mol}$ ), CDK9/cyclinT1 (rank 4, docking score: -10.4 kcal/mol), RBD2 (rank 1, docking score: $-9.4 \mathrm{kcal} / \mathrm{mol}$ ), RBD4 (rank 4, docking score: $-9.8 \mathrm{kcal} / \mathrm{mol}$ ), and both SARS-CoV-2 targets 3CLpro (rank 4, docking score: $-8.7 \mathrm{kcal} / \mathrm{mol}$ ) and PLpro (rank 10, docking score: $-9.1 \mathrm{kcal} / \mathrm{mol}$ ) (Table 2).

Structurally, CHA-378 consists of two structural moieties: a chalcone scaffold and a moiety containing sulfonamide functional group. This compound seems to be an interesting hybrid of hirsutenone (a wellknown SARS 3CLpro and PLpro inhibitor ${ }^{106}$ ) and compound A (Fig. 11). The latter was first synthesized in 2005 by Woo Duck Seo et al. as an a-glucosidase inhibitor. In their study, among all synthesized compounds, this ligand showed the best a-glucosidase inhibitory activity with $\mathrm{IC}_{50}=0.4 \mu \mathrm{M}^{107}$. Interestingly, in a recent study by Spencer J. Williams and Ethan D. Goddard-Borger in which they highlighted a-glucosidase inhibitors as host cell N-glycosylation pathway blockers with high potential for the treatment of COVID-19 108 .

In a recently published study, a large-scale screening of electrophile and non-covalent fragments was performed by Alice Douangamath et al., against the SARS-CoV-2 3CLpro through combined mass spectrometry (MS) and X-ray method ${ }^{11}$. Evaluations of this study showed that the active site of the SARS-CoV-2 3CLpro has four subsites namely S1, S1', S2 and S3. The S3 subsite has been reported to be explored and occupied by the sulfonamide group of the inhibitors and interacts with hydrogen bonding to GIn189. This space consists of His164, Met165, and Asp187, which is exposed to the solvent. So, the fragments located in this space can be oriented to the solvent. The crystal structure of 3CLpro protein bonded with compound $\mathbf{A}$ has recently been released in Protein Data Bank with PDB Code of 5RF8. This compound is able to create specific interactions in the S3 subsite. In a combined X-ray crystallography and fragment screening method, Charlie Nichols et al. reported that this fragment could interact well in the active site of the P38 MAPK target (PDB code: 5R9P) ${ }^{109}$. The presence of a structural moiety similar to this fragment (compound $\mathbf{A}$ ) in the structure of CHA-378 (Fig. 11) can be one of the important reasons for creating remarkable interactions in the active site of the p38 MAPK target (rank 10, $-9.0 \mathrm{kcal} / \mathrm{mol})$ in our screening results. Moreover, p38 MAPK was recently highlighted as one of the most promising targets for the treatment of COVID-19 ${ }^{18}$.

The sulfonamide moiety has frequently been found in the structure of both the reported 3CLpro and PLpro inhibitors. In 2008, Arun K. Ghosh and co-workers developed small molecules with potent inhibitory activity against SARS-CoV 3CLpro, some of which contain the sulfonamide functional group (Figue 11, compound C) ${ }^{110}$. In a recently published study, Armin Welker and co-workers also introduced some chalcone-amide-based small molecules with inhibitory activities against SARS-CoV/SARS-CoV-2 PLpro, which contain a sulfonamide moiety (Figue 11 , compounds $\mathbf{D}$, and $\mathbf{E}$ ) ${ }^{111}$. The mentioned experimental 
results together with our computational findings indicate that compounds CHA-297 and CHA-378 could be specific compounds with the remarkable potential to inhibit SARS-CoV-2.

The types and interacting residues for the CHA-378 and its analogue CHA-297 in the active site of PLpro and 3CLpro are displayed in Fig. 12 and Tables 7 and 8. The key interactions created by CHA-378 at the 3CLpro active site are: a conventional hydrogen bond (Leu141 with 3-OH of catechol ring), a Pi-donor Hbond (Asn142 with catechol ring), four Pi-alkyl interactions (Met49, Met165, Cys145, and His41), Pi-sigma interaction (GIn189), and two Pi-cation and Pi-Pi stacked interactions of His41 with phenyl ring of sulfonamide moiety. There is also an unfavorable acceptor-acceptor interaction of Ser144 and Leu141 with the 4-hydroxy group of the catechol moiety. This unfavorable interaction is distracting in the active site of the $3 \mathrm{CL}$ ro and reduces its binding energy in the gas phase (Table $3, \Delta \mathrm{G}_{\text {gas }}$ ). Finding that presence of the two hydroxy groups in the catechol moiety is not accepted, hence the existence of only one hydroxyl group by selecting the most similar CHA-297 in our compound library should be a rational decision.

As shown in Table 3, the MM/PB(GB)SA results indicated that the affinity of CHA-297 is higher than CHA378 by 6.5 and $3.8 \mathrm{kcal} / \mathrm{mol}$ calculated by the GB and PB respectively towards the 3CLpro active site. Investigating the mode of binding of CHA-297 to the 3CLpro active site showed that the key hydroxyl group discussed earlier has formed a hydrogen bond with Asn142 without any unfavorable interactions (Fig. 12). Interestingly, it was found that the sulfonamide moiety occupied the S3 subsite and hydrogen bonded to Gln189 which has been proved by a recent study to be a specific site for the binding of the sulfonamide containing 3CLpro inhibitors ${ }^{11}$.

CHA-378 and CHA-297 were also investigated in the active site of the PLpro since the primary results of the docking screening indicated them as PLpro inhibitors as well (Table 2). In summary, $\mathrm{NH}$ of the sulfonamide moieties in both CHA-378 and CHA-297 creates hydrogen bond with Glu167 in the active site of the PLpro. On the other hand, oxygen atoms of the sulfonamide moiety of CHA-297 formed hydrogen bond interactions with Gln269. Similarly, Gln269 and Leu162 residues formed the same interactions Pisigma (GIn269) and amide-Pi stacked (Leu162) with the A-ring of the chalcone backbone in both CHA297 and CHA-378. Tyr264 is another key residue that creates the same interactions in the PLpro active site for both CHA-297 and CHA-378 including a conventional hydrogen bond with the carbonyl group of the chalcone moiety, and a Pi-Pi T-shaped interaction with the catechol and phenolic rings of CHA-378 and CHA-297 respectively. However, the results of the MM/PB(GB)SA calculations indicated that these two compounds are weak PLpro inhibitors and can not be considered as PLpro hit compounds for further study.

\section{Active ligands selective towards the 3CLpro}

The biphenyl urea scaffold is a recently identified fragment introduced by Alice Douangamath and coworkers that can generate specific interactions in the active site of the SARS-CoV-2 3CLpro enzyme ${ }^{11}$. As the researchers well characterized, the $\mathrm{S} 1$ subsite of the 3CLpro-active site revealed the two key residues His163, which is interacting with the pyridine nitrogen or with nitrogen-containing heterocycles, and 
Glu166 which is a key amino acid in the formation of hydrogen bonds to the amide or ureide substructures of the 3CLpro-inhibitors. In Fig. 13, the chemical structure of some of the biaryl urea small molecules (compounds $\mathbf{B}$ and $\mathbf{E}$ ) and their isosteric amide-analogues (compounds $\mathbf{F}$ and $\mathbf{G}$ ) with their corresponding PDB codes which were recently released as selective 3CLpro inhibitors are displayed. Also, the structural similarity of the reported biaryl urea compounds with some of our ureide-chalcone hybrid structures existed in our library were found to be as hit compounds selective against 3CLpro (see Table 2) are displayed in Fig. 13.

The key interactions of the co-crystal compound $\mathbf{B}$ in the active site of the 3CLpro (PDB code 5R83) are shown in Fig. 14A. Correspondingly, $\mathrm{NH}$ and oxygen atoms of the ureide moiety in compound $\mathbf{B}$ form two important conventional hydrogen bonds with the residues Arg188 and Glu166. Investigating the binding mode of the ureide-chalcone hybrid structures CHA-233, CHA-236, CHA-383, CHA-384, CHA-392, and CHA397 and interacting residues within the 3CLpro active site are shown in Fig. 14B. As discussed earlier, these compounds were previously synthesized and reported as antimalarial agents ${ }^{112}$. The spatial orientation of these ureide-chalcones in the active site of the $3 \mathrm{CL}$ pro is precisely the same and the ureide substructure formed two conventional hydrogen bonds by the interaction of Arg188 and the two $\mathrm{NH}$ atoms (Fig. 14B). These hydrogen bonding interactions cause the urea to be oriented exactly at a specific location adjacent to the Arg188 (Fig. 15). These ureide-chalcone hybrid structures have a high selectivity to 3 CLpro compared to PLpro, and significantly produce stronger interactions than compound $\mathbf{B}$ (as standard) at the SARS-CoV-2 3CLpro active site.

Among the evaluated 3CLpro-selective ureide-chalcones, CHA-384 generates the highest affinities in the active site of the virus and host-based targets compared to the other congeners. The rankings predicted for CHA-384 against the different virus and host-based targets (see Table 2) is as follows: CDK2/CyclinA (rank 7, docking score: $-9.2 \mathrm{kcal} / \mathrm{mol}$ ), ERK2 (rank 2, docking score: $-9.9 \mathrm{kcal} / \mathrm{mol}$ ), DHODH (rank 2, docking score: $-12.7 \mathrm{kcal} / \mathrm{mol}$ ), BRD4 (rank 7, docking score: -9.7 kcal/mol), sigma-1 receptor (rank 3 , docking score: $-11.6 \mathrm{kcal} / \mathrm{mol}$ ), and 3CLpro (rank 2, docking score: $-8.9 \mathrm{kcal} / \mathrm{mol}$ ). In addition to the conventional hydrogen bonds mentioned above, the most important interactions of CHA-384 in the active site of the 3CLpro are as follows: Pi-Alkyl interactions of Met49 and Met165 residues with A-phenyl ring of the chalcone backbone, and Pi-Alkyl interaction of Pro168 with phenyl ring of the ureide moiety.

Moreover, the chlorine atom of the ureide moiety generates two alkyl interactions with Pro168 and Ala191 residues. The Pi-sigma interaction of the B-phenyl ring with Thr25, and a Pi-Pi Stacked interaction of the A-phenyl ring with His41 are other interactions formed by CHA-384 in the active site of the 3CLpro. These interactions are shown comparatively for CHA-384 and its congeners in Fig. 14B.

\section{Selective ligands towards the SARS-CoV-2 PLpro}

A long time after the emergence of the SARS-CoV pandemic in 2002, structure-based design approaches for the development of PLpro inhibitors were unsuccessful due to the insufficiency of the target structural information. For the first time in 2008, Ghosh and co-workers were able to identify two selective SARSCoV PLpro inhibitors by performing an extensive high throughput screening on 50,080 compounds ${ }^{113}$. 
Interestingly, GRL0617 (Fig. 16A) with a chalcone-amide backbone is a well-known compound generated from the study of Ghosh et al, and currently it is used for the design and development of the SARS-

CoV/SARS-CoV-2 PLpro inhibitors as a standard and lead compound. Herein, to validate the assessments and better understanding the interactions, GRL0617 was used as a standard.

Interestingly, CHA-7, CHA-11, CHA-37, CHA-44, and CHA-177 showed superior docking scores among our chlacone-based compound library against the SARS-CoV-2 PLpro and host-based targets, and also CHA37 was found to have the highest affinity towards the PLpro among them (Table 2). The ranking and docking energy score of CHA-37 at the active sites of the host and viral-based targets are as follows: p38 MAPK (rank 2, -9.4 kcal/mol), Cathepsin L (rank 4, -8.3 kcal/mol), CDK1 (rank 3, -9.3 kcal/mol), CDK2/CyclinA (rank 9, -9.6 kcal/mol), CDK9/cyclinT1 (rank 7, -9.9 kcal/mol), ERK2 (rank 4, -9.5 kcal/mol), DHODH (rank 9, -12.0 kcal/mol), CK2 alpha' (rank 1, -11.4 kcal/mol), BRD2 (rank 5, -8.8 kcal/mol), BRD4 (rank 1, -10.4 kcal/mol), and sigma-1 receptor (rank 2, -11.7 kcal/mol), 3CLpro (rank 12, $-8.3 \mathrm{kcal} / \mathrm{mol}$ ) and PLpro (rank $3,-9.5 \mathrm{kcal} / \mathrm{mol}$ ). Although, the calculated binding affinity of CHA-37 in the active site of the PLpro was calculated to be lower than GRL0617 by the PB and GB methods, it still shows more affinity than the co-crystal ligand of the 7JN2 PDB code (see Table 4). Interestingly, its calculated binding free energy of $-19.20 \mathrm{kcal} / \mathrm{mol}$ by the PB method indicated its higher affinity towards the PLpro compared to the standard inhibitor hisutenone $(-16.52 \mathrm{kcal} / \mathrm{mol})$.

As displayed in Fig. 16B and Table 8, CHA-37 created important interactions in the active site of the PLpro. The benzotriazole moiety of this compound forms key interactions, including Pi-alkyl with Pro247 and Pro248 residues, one amide-Pi stacked interaction resulting from the interaction of triazole fraction with Asn267, and one Pi-Pi T-Shaped interaction with Tyr268.

Similarly, the naphthyl ring of the GRL0617 creates three Pi-alkyl interactions with Pro247 and Pro248 residues, and also two Pi-Pi T-shaped interactions with Tyr268. In addition, the formation of three conventional hydrogen bonds by the carbonyl group with Tyr268, amide-NH with Asp164, and the amino group of GRL0617 with Gly163, help to strengthen the binding of this compound in the active site of the SARS-CoV-2 PLpro. 2D and 3D interactions of CHA-37 and GRL0617 in the active site of the SARS-CoV-2 PLpro enzyme are shown in Fig. 16.

Structural evaluation of the selective PLpro ligands shows outstanding similarities. In general, these selective PLpro compounds have unsubstituted and flat nitrogen-containing aromatic groups on ring $B$ of the chalcone backbone (Fig. 17A and 17B). Correspondingly, quinoxaline ring in CHA-11, benzo-[1, 2, 3]triazole in CHA-37, and benzimidazole in CHA-44 in position 2 or 3 on the B-ring are a main motif for binding. As indicated in the structure of GRL0617, the same position is occupied by a fused phenyl ring with the B-ring of the chalcone backbone. Interestingly, in a recent structure-based drug design attempt, extensive SAR evaluations have led to a chalcone-amide isoster bearing a 2-thienyl at position 3 of ring $B$ for producing the strongest derivatives against SARS-CoV-2 PLpro (Fig. 17A, structure H) ${ }^{105}$. The metaand para-fluorophenyl substitutions in positions 2 and 3 of the B-ring also existed in the structure of CHA7 and CHA-177 which were found to have high docking scores in our compound library. These 
substitutions are also found in the structure of high affinity SARS-CoV PLpro inhibitors ever reported which confirms and validates our results (Fig. 17C, compounds $L$ and $M)^{114}$. Therefore, a fused and nitrogen containing aromatic ring or a fluorophenyl ring substituted at position 3 of the chalcone B-ring (or chalcone-amide) is useful for increasing the inhibitory effect of compounds against the PLpro enzyme.

Tables 7 and 8 comparatively summarize all the predicted interactions related to the most promising CHA-12, CHA-37, CHA-378, and CHA-384 along with the appropriate standards baicalein and GRL0617 in the active site of the SARS-CoV-2 3CLpro and PLpro.

In addition to having strong affinity of CHA-37 in the PLpro active site (rank 3, docking score $=-9.5$ $\mathrm{kcal} / \mathrm{mol})$, it is interesting that this ligand also creates remarkable interactions in the 3CLpro active site with the rank of 12 among the 757 chalcone-based compound library. Accordingly, we examined also the interactions of this compound in the active site of both the viral targets PLpro and 3CLpro.

\section{Benzotriazole for selective 3CLpro inhibition}

One of the important reasons for creating strong interactions of CHA-37 in the 3CLpro active site could also be attributed to the presence of the benzotriazole group. This moiety has been observed in the structure of the previously reported potent 3CLpro inhibitors, as in representative compounds $\mathbf{I}, \mathbf{J}$, and $\mathbf{K}$ (Fig. 17) ${ }^{115,116 .}$

Comparison of the interactions of CHA-37 with compound $\mathbf{J}$, a highly potent SARS-CoV 3CLpro inhibitor $\left(\mathrm{IC}_{50}=51 \mathrm{nM}\right)$, in the SARS-CoV-2 3CLpro active site shows that benzotriazole moieties of both compounds are occupying exactly the same space, and mostly interact with the same residues His41, Cys44, and Met49 (Fig. 19). The strong affinity of CHA-37 towards the 3CLpro binding space was further confirmed by the MM/PB(GB)SA calculations (Fig. 5 and Table 3). The results showed that CHA-37 has higher affinity towards the $3 C L$ pro active site than the standard inhibitors baicalein $(-30.93 \mathrm{kcal} / \mathrm{mol})$ and hirsutenone $(-24.12 \mathrm{kcal} / \mathrm{mol})$ which was evidenced by its binding free energy of $-31.09 \mathrm{kcal} / \mathrm{mol}$ in the GB method.

\section{Conclusion}

Since the 3CLpro and PLpro play a key role in the replication of SARS-CoV-2, these enzymes are the main therapeutic targets for the treatment of COVID-19. In addition, modulators of the host-based targets which have roles in the virus life cycle can assist for the suppression of the SARS-CoV-2. In this study, a targeted and extensive computational evaluation was performed to investigate the affinity of 757 chalcone-based structures in inhibition of the SARS-CoV-2 3CLpro and PLpro enzymes, and modulation of a wide range of host-based targets. Our results indicated that the ureide substructure is an integral part for the potent and selective 3CLpro inhibition by hydrogen bonding to Arg188 and occupying the S1 subsite.

Correspondingly, the sulfonamide moiety of CHA-297 can infer 3CLpro inhibition potential to some extent by occupying the $S 3$ subsite and interacting with GIn189. CHA-384 as a high affinity and a selective 
3CLpro inhibitor was introduced as a lead compound in our study to inhibit 3CLpro. Benzotriazole fragment was also found as a well-interacting residue in the binding space of the 3CLpro and PLpro enzymes which is evidenced by the introduction of CHA-37. These findings are in excellent accordance with the results of a recent large-scale fragment-based screening performed by mass spectrometry and Xray crystallography. The stablished relaxant effects of CHA-12 on pulmonary smooth muscles through antagonistic activity on CysLT1 which was reported previously by in vivo and in vitro assessments, along with our predictions of its amazing effects on all virus and host-based targets promise the introduction of a valuable compound for the treatment of COVID-19.

\section{Methods}

\section{Compound library preparation}

PubChem database was searched by keyword "Chalcone" and after applying the lipinski's rule of 5, the number of 757 chalcone-based structures were retrieved, named as CHA-1 to CHA-757 and used in this study (See Supplementary Material for the mol2 format of our 757 compound library).

\section{Target selection and preparation}

Firstly, the structure of virus-based targets 3CLPro (PDB code: 6M2N) and PLPro (PDB code: 7JN2), and various host-based targets including: p38 MAPK (PDB code: 4EH3), Cathepsin L (PDB code: 5MQY), CDK1 (PDB code: 6GU2), CDK2/CyclinA (PDB code: 6GUB), CDK9/cyclinT1 (PDB code: 3BLR), ERK2 (PDB code: 3SA0), HDAC2 (PDB code: 4LXZ), DHODH (PDB code: 5ZF7), CK2 alpha' (PDB code: 5M4U), BRD2 (PDB code: 4J1P), BRD4 (PDB code: 6HOV), and sigma-1 receptor (PDB code: 6DK1) were extracted from the Protein Data Bank (www.rcsb.org) in PDB format. The preparation steps of proteins and ligands were performed based on the method described in our recent study.

The selected host-based and virus-based targets with the associated PDB codes as well as the basic parameters of the docking protocol are summarized in Table 9.

\section{Virtual screening}

A python script for performing virtual screening by AutoDock Vina and ranking the bound conformations based on their best binding energy was used for docking our 757 chalcone-based compound library against the 14 selected virus and host-based targets. The center and size of the grid box in each docking course has been presented in Table $3^{117}$.

\section{Molecular dynamics simulation \& MM/PB(GB)SA calculations}

GROMACS version 2020 installed on Ubuntu 20.04 Linux workstation was used for dynamics simulation of compounds in the binding site in explicit water model TIP3P and Amber99SB-ILDN force field was used for topology generation. Topology of ligands were generated by ACPYPE and GAFF was used for 
ligand topology generation. Ligand atomic charges were calculated using PM3 in Gaussian 09. The topology and coordinate files for the protein were generated using pdb2gmx program of GROMACS package taking parameters from the Amber99SB-ILDN force field. The coordinate and topology files of the protein and the ligands were then merged to obtain the final starting structure and topology file for each complex.

The complex was centered in a dodecahedron periodic box and solvated by the addition of water molecules (Simple point charge model). The total charge of the system was then neutralized by addition of sodium and chloride ions as required. Sequentially, energy minimization was performed by steepest descent algorithm. The system was then gradually heated to $300 \mathrm{~K}$ and was equilibrated $100 \mathrm{ps}$ using the NVT (constant volume and temperature) ensemble with position restraint for the heavy atoms followed by 100 ps equilibration in the NPT (constant pressure and temperature) ensemble at $1 \mathrm{~atm}$. Both temperature and pressure were regulated using the Berendsen algorithm. Finally, the full system was subjected to $10 \mathrm{~ns}$ MD simulation with a $2 \mathrm{fs}$ time step interval. The temperature and pressure were maintained at $300 \mathrm{~K}$ and $1 \mathrm{~atm}$ using the v-rescale temperature and Parrinello-Rahman pressure coupling method. The short-range non-bonded interactions were computed for the atom pairs within the cut-off of $1.2 \mathrm{~nm}$, while the long-range electrostatic interactions were calculated using Particle-Mesh-Ewald summation method with fourth-order cubic interpolation and $1.2 \AA$ grid spacing. All h-bonds were constrained using the parallel LINCS method.

Finally, Molecular Mechamics/Poisson Boltzmann (or Generalized Born) Surface Area MM/PB(GB)SA method for calculating free energy of binding was accomplished by gmx_MMPBSA 1.4.2 introduced by Mario S. Valdés Tresanco ${ }^{118}$. gmx_MMPBSA is a new tool based on AMBER's MMPBSA.py in AmberTools20 aiming to perform end-state free energy calculations with GROMACS files.

Molecular Mechanics / Poisson Boltzmann (or Generalized Born) Surface Area (MM/PB(GB)SA) is a postprocessing method in which representative snapshots from an ensemble of conformations are used to calculate the free energy change between a bound and unbound state of a ligand in a receptor. Free energy differences are calculated by combining the so-called gas phase energy contributions ( $\mathrm{MM}$ term) that are independent of the chosen solvent model as well as solvation free energy components (electrostatic and hydrophobic) calculated from an implicit solvent model Poisson Boltzmann or Generalized Born for each species. Entropy contributions $(-T \Delta S)$ to the total free energy was added using the so-called Interaction Entropy method, which is theoretically rigorous, computationally efficient, and numerically reliable for calculating entropic contribution to free energy in protein-ligand binding and other interaction processes.

The gas phase free energy contributions were calculated by mmpbsa_py_energy (use_sander $=0$ ) within the AmberTools package. The PB equation was solved numerically by the pbsa program included with AmberTools20 (sander_apbs $=0$ ). Hydrophobic contribution in GB was approximated by the LCPO method (molsurf $=0$ ) implemented within sander and the total non-polar solvation free energy in PB was modeled as a single term linearly proportional to the solvent accessible surface area (inp $=1$ ). 
The following parameters were used for the calculation of binding energies by MM/PB(GB)SA method. Start frame $=900$, end frame $=1000$, interval $=20$, entropy $=$ interaction entropy, igb $=5$, saltcon $=0.150$, inp $=1$, istrng $=0.150$, fillratio $=4.0$, radiopt $=0$.

The following formulas represent the calculation of the parameters and energy components.

$\Delta \mathrm{E}=\mathrm{E}_{\text {complex }}-\mathrm{E}_{\text {receptor }}-\mathrm{E}_{\text {ligand }}$

$\Delta \mathrm{G}_{\mathrm{gas}}=\Delta \mathrm{G}_{\mathrm{VDW}}+\Delta \mathrm{G}_{\mathrm{EEL}}$

For the GB method: $\Delta \mathrm{G}_{\text {solv }}=\Delta \mathrm{G}_{\mathrm{EGB}}+\Delta \mathrm{G}_{\mathrm{ESURF}}$

For the PB method: $\Delta \mathrm{G}_{\text {solv }}=\Delta \mathrm{G}_{\mathrm{EPB}}+\Delta \mathrm{G}_{\mathrm{ENPOLAR}}$

$\Delta G_{\text {total }}=\Delta G_{\text {gas }}+\Delta G_{\text {solv }}$

$\Delta \mathrm{G}_{\mathrm{b}}=\Delta \mathrm{G}_{\text {total }}+\mathrm{IE}$

\section{Druglikeness \& Pharmacokinetic prediction}

Lipinski's rule of 5 and violatons were calculated by PreADME, BBB permeation and GI absorbtion were estimated by swisSADME and P-glycoprotein inhibition, acute oral toxicity, carcinogenicity were calculated by admetSAR web-based servers.

\section{Abbreviations}




\begin{tabular}{|lll|}
\hline 1 & COVID-19 & Coronavirus disease-2019 \\
\hline 2 & SARS-CoV & Severe Acute Respiratory Syndrome Coronavirus \\
\hline 3 & SARS-CoV-2 & Severe Acute Respiratory Syndrome Coronavirus 2 \\
\hline 4 & MERS-CoV & Middle East Respiratory Syndrome \\
\hline 5 & 3CLpro & 3-Chymotrypsin-Like Protease \\
\hline 7 & PLpro & Papain-Like Protease \\
\hline 8 & DAATs & Host-Based Antiviral Targets \\
\hline 9 & MM/PB(GB)SA & Molecular Mechamics/Poisson Boltzmann (Generalized Born)Surface Area \\
\hline 10 & NSPs & Non-Structural Proteins \\
\hline 11 & SPs & Structural Proteins \\
\hline 12 & ACE2 & Angiotensin-Converting Enzyme II \\
\hline 13 & p38 MAPK & p38 Mitogen-Activated Protein Kinase \\
\hline 14 & CDKs & Cyclin-Dependent Kinases \\
\hline 15 & DHODH & Dihydrooroate dehydrogenase \\
\hline 16 & HDAC2 & Histone deacetylase 2 \\
\hline 17 & BRDs & Bromodomain-containing proteins \\
\hline
\end{tabular}

\section{Declarations}

\section{Acknoledgements}

This work was supported by a grant from Mazandaran University of Medical Sciences (MAZUMS) through grant number 10282 .

\section{Author contributions}

M.V. contributed in the computational and molecular modeling of the study and writing the manuscript. Supervision of the study, interpretation of the results and writing the manuscript was done by H.I.

\section{Competing interests}

The authors declare no competing interests.

\section{References}


1. Lu, R., et al. Genomic characterisation and epidemiology of 2019 novel coronavirus: implications for virus origins and receptor binding. The lancet395, 565-574 (2020).

2. Mukhtar, M., et al. Antiviral potentials of medicinal plants. Virus research131, 111-120 (2008).

3. Li, S.-y., et al. Identification of natural compounds with antiviral activities against SARS-associated coronavirus. Antiviral research67, 18-23 (2005).

4. Benarba, B. \& Pandiella, A. Medicinal plants as sources of active molecules against COVID-19. Frontiers in Pharmacology11(2020).

5. Verma, S., et al. Anti-SARS-CoV natural products with the potential to inhibit SARS-CoV-2 (COVID-19). Frontiers in Pharmacology11, 1514 (2020).

6. Kim, D., et al. The architecture of SARS-CoV-2 transcriptome. Cel/181, 914-921. e910 (2020).

7. Yao, H., et al. Molecular architecture of the SARS-CoV-2 virus. Cel/183, 730-738. e713 (2020).

8. Ferreira, J.C. \& Rabeh, W.M. Biochemical and biophysical characterization of the main protease, 3chymotrypsin-like protease (3CLpro) from the novel coronavirus SARS-CoV 2. Scientific reports10, 110 (2020).

9. Jin, Z., et al. Structure of M pro from SARS-CoV-2 and discovery of its inhibitors. Nature582, 289-293 (2020).

10. Shin, D., et al. Papain-like protease regulates SARS-CoV-2 viral spread and innate immunity. Nature587, 657-662 (2020).

11. Douangamath, A., et al. Crystallographic and electrophilic fragment screening of the SARS-CoV-2 main protease. Nature communications11, 1-11 (2020).

12. Richman, D.D. Antiviral drug resistance. Antiviral research71, 117-121 (2006).

13. Strasfeld, L. \& Chou, S. Antiviral drug resistance: mechanisms and clinical implications. Infectious Disease Clinics24, 809-833 (2010).

14. de Chassey, B., Meyniel-Schicklin, L., Vonderscher, J., André, P. \& Lotteau, V. Virus-host interactomics: new insights and opportunities for antiviral drug discovery. Genome medicine6, 1-14 (2014).

15. Wang, Q., et al. Structural and functional basis of SARS-CoV-2 entry by using human ACE2. Cel/181, 894-904. e899 (2020).

16. Hoffmann, M., et al. SARS-CoV-2 cell entry depends on ACE2 and TMPRSS2 and is blocked by a clinically proven protease inhibitor. cel/181, 271-280. e278 (2020).

17. Sacco, M.D., et al. Structure and inhibition of the SARS-CoV-2 main protease reveal strategy for developing dual inhibitors against Mpro and cathepsin L. Science Advances6, eabe0751 (2020).

18. Grimes, J.M. \& Grimes, K.V. p38 MAPK inhibition: A promising therapeutic approach for COVID-19. Journal of Molecular and Cellular Cardiology144, 63-65 (2020).

19. Gutierrez-Chamorro, L., Felip, E., Ezeonwumelu, I.J., Margelí, M. \& Ballana, E. Cyclin-dependent Kinases as Emerging Targets for Developing Novel Antiviral Therapeutics. Trends in Microbiology (2021). 
20. Xu, Y. \& Jiang, H. Potential treatment of COVID-19 by inhibitors of human dihydroorotate dehydrogenase. Protein \& Cel/11, 699-702 (2020).

21. Park, J.-Y., et al. Chalcones isolated from Angelica keiskei inhibit cysteine proteases of SARS-CoV. Journal of enzyme inhibition and medicinal chemistry31, 23-30 (2016).

22. Park, J.-Y., et al. Evaluation of polyphenols from Broussonetia papyrifera as coronavirus protease inhibitors. Journal of enzyme inhibition and medicinal chemistry32, 504-512 (2017).

23. Kim, D.W., et al. Phenolic phytochemical displaying SARS-CoV papain-like protease inhibition from the seeds of Psoralea corylifolia. Journal of enzyme inhibition and medicinal chemistry29, 59-63 (2014).

24. Jo, S., Kim, H., Kim, S., Shin, D.H. \& Kim, M.S. Characteristics of flavonoids as potent MERS-CoV 3Clike protease inhibitors. Chemical biology \& drug design94, 2023-2030 (2019).

25. Elkhalifa, D., Al-Hashimi, I., Al Moustafa, A.-E. \& Khalil, A. A comprehensive review on the antiviral activities of chalcones. Journal of Drug Targeting, 1-17 (2020).

26. Kopecky-Bromberg, S.A., Martinez-Sobrido, L. \& Palese, P. 7a protein of severe acute respiratory syndrome coronavirus inhibits cellular protein synthesis and activates p38 mitogen-activated protein kinase. Journal of virology80, 785-793 (2006).

27. Marchant, D., et al. Toll-like receptor 4-mediated activation of p38 mitogen-activated protein kinase is a determinant of respiratory virus entry and tropism. Journal of virology84, 11359-11373 (2010).

28. Mizutani, T., Fukushi, S., Saijo, M., Kurane, I. \& Morikawa, S. Phosphorylation of p38 MAPK and its downstream targets in SARS coronavirus-infected cells. Biochemical and biophysical research communications319, 1228-1234 (2004).

29. Kono, M., et al. Inhibition of human coronavirus $229 \mathrm{E}$ infection in human epithelial lung cells (L132) by chloroquine: involvement of p38 MAPK and ERK. Antiviral research77, 150-152 (2008).

30. Banerjee, S., Narayanan, K., Mizutani, T. \& Makino, S. Murine coronavirus replication-induced p38 mitogen-activated protein kinase activation promotes interleukin-6 production and virus replication in cultured cells. Journal of virology76, 5937-5948 (2002).

31. Padhan, K., Minakshi, R., Towheed, M.A.B. \& Jameel, S. Severe acute respiratory syndrome coronavirus 3 a protein activates the mitochondrial death pathway through p38 MAP kinase activation. Journal of general virology89, 1960-1969 (2008).

32. McCaskill, J.L., et al. Broad-spectrum inhibition of respiratory virus infection by microRNA mimics targeting p38 MAPK signaling. Molecular Therapy-Nucleic Acids7, 256-266 (2017).

33. Liu, T., Luo, S., Libby, P. \& Shi, G.-P. Cathepsin L-selective inhibitors: A potentially promising treatment for COVID-19 patients. Pharmacology \& therapeutics, 107587 (2020).

34. Huang, I.-C., et al. SARS coronavirus, but not human coronavirus NL63, utilizes cathepsin L to infect ACE2-expressing cells. Journal of Biological Chemistry281, 3198-3203 (2006).

35. Simmons, G., et al. Inhibitors of cathepsin L prevent severe acute respiratory syndrome coronavirus entry. Proceedings of the National Academy of Sciences102, 11876-11881 (2005). 
36. Zhao, M.-M., et al. Cathepsin L plays a key role in SARS-CoV-2 infection in humans and humanized mice and is a promising target for new drug development. Signal transduction and targeted therapy6, 1-12 (2021).

37. Bosch, B.J., Bartelink, W. \& Rottier, P.J. Cathepsin L functionally cleaves the severe acute respiratory syndrome coronavirus class I fusion protein upstream of rather than adjacent to the fusion peptide. Journal of virology82, 8887-8890 (2008).

38. Ferraro, F., et al. Identification of chalcones as Fasciola hepatica cathepsin L inhibitors using a comprehensive experimental and computational approach. PLoS neglected tropical diseases10, e0004834 (2016).

39. Garg, S. \& Raghav, N. Inhibitory potential of some chalcones on cathepsins B, H and L. RSC advances5, 72937-72949 (2015).

40. Surjit, M., Liu, B., Chow, V.T. \& Lal, S.K. The nucleocapsid protein of severe acute respiratory syndrome-coronavirus inhibits the activity of cyclin-cyclin-dependent kinase complex and blocks $\mathrm{S}$ phase progression in mammalian cells. Journal of Biological Chemistry281, 10669-10681 (2006).

41. Schang, L.M. Cyclin-dependent kinases as cellular targets for antiviral drugs. Journal of Antimicrobial Chemotherapy50, 779-792 (2002).

42. Gargouri, M., Alzwi, A. \& Abobaker, A. Cyclin dependent kinase inhibitors as a new potential therapeutic option in management of COVID-19. Medical Hypotheses146, 110380 (2021).

43. Heaton, B.E., et al. SRSF protein kinases 1 and 2 are essential host factors for human coronaviruses including SARS-CoV-2. BioRxiv (2020).

44. Cribier, A., Descours, B., Valadão, A.L.C., Laguette, N. \& Benkirane, M. Phosphorylation of SAMHD1 by cyclin A2/CDK1 regulates its restriction activity toward HIV-1. Cell reports3, 1036-1043 (2013).

45. Ludgate, L., et al. Cyclin-dependent kinase 2 phosphorylates s/tp sites in the hepadnavirus core protein C-terminal domain and is incorporated into viral capsids. Journal of virology86, 12237-12250 (2012).

46. He, W., Staples, D., Smith, C. \& Fisher, C. Direct activation of cyclin-dependent kinase 2 by human papillomavirus E7. Journal of virology77, 10566-10574 (2003).

47. Bouchard, M., Giannakopoulos, S., Wang, E.H., Tanese, N. \& Schneider, R.J. Hepatitis B virus HBx protein activation of cyclin A-cyclin-dependent kinase 2 complexes and G1 transit via a Src kinase pathway. Journal of virology75, 4247-4257 (2001).

48. Wang, S. \& Fischer, P.M. Cyclin-dependent kinase 9: a key transcriptional regulator and potential drug target in oncology, virology and cardiology. Trends in pharmacological sciences29, 302-313 (2008).

49. Khan, S.Z. \& Mitra, D. Cyclin K inhibits HIV-1 gene expression and replication by interfering with cyclin-dependent kinase 9 (CDK9)-cyclin T1 interaction in Nef-dependent manner. Journal of Biological Chemistry286, 22943-22954 (2011).

50. Zhang, J., Li, G. \& Ye, X. Cyclin T1/CDK9 interacts with influenza A virus polymerase and facilitates its association with cellular RNA polymerase II. Journal of virology84, 12619-12627 (2010). 
51. Klebl, B.M. \& Choidas, A. CDK9/cyclin T1: a host cell target for antiretroviral therapy. (2006).

52. Kindrachuk, J., et al. Antiviral potential of ERK/MAPK and PI3K/AKT/mTOR signaling modulation for Middle East respiratory syndrome coronavirus infection as identified by temporal kinome analysis. Antimicrobial agents and chemotherapy59, 1088-1099 (2015).

53. Varshney, B. \& Lal, S.K. SARS-CoV accessory protein 3b induces AP-1 transcriptional activity through activation of JNK and ERK pathways. Biochemistry50, 5419-5425 (2011).

54. Johnson, T.R., McLellan, J.S. \& Graham, B.S. Respiratory syncytial virus glycoprotein G interacts with DC-SIGN and L-SIGN to activate ERK1 and ERK2. Journal of virology86, 1339-1347 (2012).

55. Chen, W., Monick, M.M., Carter, A.B. \& Hunninghake, G.W. Activation of ERK2 by respiratory syncytial virus in A549 cells is linked to the production of interleukin 8. Experimental lung research26, 13-26 (2000).

56. Martin, S., Harris, D.T. \& Shisler, J. The C11R gene, which encodes the vaccinia virus growth factor, is partially responsible for MVA-induced NF-KB and ERK2 activation. Journal of virology86, 9629-9639 (2012).

57. Takahashi, Y., et al. Histone deacetylase inhibitors suppress ACE2 and ABO simultaneously, suggesting a preventive potential against COVID-19. Scientific Reports11, 1-9 (2021).

58. Murthy, P.K., Sivashanmugam, K., Kandasamy, M., Subbiah, R. \& Ravikumar, V. Repurposing of histone deacetylase inhibitors: A promising strategy to combat pulmonary fibrosis promoted by TGF$\beta$ signalling in COVID-19 survivors. Life sciences, 118883 (2020).

59. Sivashanmugam, K., Kandasamy, M., Subbiah, R. \& Ravikumar, V. Repurposing of histone deacetylase inhibitors: A promising strategy to combat pulmonary fibrosis promoted by TGF- $\beta$ signalling in COVID-19 survivors. Life Sci, 118883-118883 (2021).

60. Mohamed, M.F., et al. Design, synthesis, docking studies and biological evaluation of novel chalcone derivatives as potential histone deacetylase inhibitors. Bioorganic chemistry72, $32-41$ (2017).

61. Orlikova, B., et al. Natural chalcones as dual inhibitors of HDACs and NF-kB. Oncology reports28, 797-805 (2012).

62. Coelho, A.R. \& Oliveira, P.J. Dihydroorotate dehydrogenase inhibitors in SARS-CoV-2 infection. (Wiley Online Library, 2020).

63. Berber, B. \& Doluca, O. A comprehensive drug repurposing study for COVID19 treatment: novel putative dihydroorotate dehydrogenase inhibitors show association to serotonin-dopamine receptors. Briefings in bioinformatics (2021).

64. Luban, J., et al. The DHODH inhibitor PTC299 arrests SARS-CoV-2 replication and suppresses induction of inflammatory cytokines. Virus research292, 198246 (2021).

65. Xiong, R., et al. Novel and potent inhibitors targeting DHODH are broad-spectrum antivirals against RNA viruses including newly-emerged coronavirus SARS-CoV-2. Protein \& cel/11, 723-739 (2020).

66. Xiong, R., et al. Novel and potent inhibitors targeting DHODH, a rate-limiting enzyme in de novo pyrimidine biosynthesis, are broad-spectrum antiviral against RNA viruses including newly emerged 
coronavirus SARS-CoV-2. BioRxiv (2020).

67. Hahn, F., et al. IMU-838, a Developmental DHODH Inhibitor in Phase II for Autoimmune Disease, Shows Anti-SARS-CoV-2 and Broad-Spectrum Antiviral Efficacy In Vitro. Viruses12, 1394 (2020).

68. Calistri, A., et al. The new generation hDHODH inhibitor MEDS433 hinders the in vitro replication of SARS-CoV-2. bioRxiv (2020).

69. Xia, C., et al. Casein kinase 1a mediates the degradation of receptors for type I and type II interferons caused by hemagglutinin of influenza A virus. Journal of virology92(2018).

70. Quintavalle, M., et al. Hepatitis C virus NS5A is a direct substrate of casein kinase I-a, a cellular kinase identified by inhibitor affinity chromatography using specific NS5A hyperphosphorylation inhibitors. Journal of Biological Chemistry282, 5536-5544 (2007).

71. Bhattacharya, D., Ansari, I.H. \& Striker, R. The flaviviral methyltransferase is a substrate of Casein Kinase 1. Virus research141, 101-104 (2009).

72. Bhargavan, B. \& Kanmogne, G.D. Epigenetics, N-myrystoyltransferase-1 and casein kinase-2-alpha modulates the increased replication of HIV-1 CRF02_AG, compared to subtype-B viruses. Scientific reports9, 1-19 (2019).

73. Zhang, L., et al. The down-regulation of casein kinase 1 alpha as a host defense response against infectious bursal disease virus infection. Virology512, 211-221 (2017).

74. Campagna, M., et al. Impaired hyperphosphorylation of rotavirus NSP5 in cells depleted of casein kinase $1 \mathrm{a}$ is associated with the formation of viroplasms with altered morphology and a moderate decrease in virus replication. Journal of General Virology88, 2800-2810 (2007).

75. Chlamydas, S., Papavassiliou, A.G. \& Piperi, C. Epigenetic mechanisms regulating COVID-19 infection. Epigenetics16, 263-270 (2021).

76. Tian, R., et al. BRD2 inhibition blocks SARS-CoV-2 infection in vitro by reducing transcription of the host cell receptor ACE2. bioRxiv (2021).

77. O'Meara, M.J., Guo, J.Z., Swaney, D.L., Tummino, T.A. \& Hüttenhain, R. A SARS-CoV-2-human proteinprotein interaction map reveals drug targets and potential drug-repurposing. BioRxiv (2020).

78. Acharya, A., et al. Highly potent PI3K-a/mTOR/BRD4 inhibitor for the targeted inhibition of SARSCoV-2. bioRxiv (2021).

79. Acharya, A., et al. Blockade of SARS-CoV-2 infection in-vitro by highly potent PI3K-a/mTOR/BRD4 inhibitor. bioRxiv (2021).

80. Hashimoto, K. Repurposing of CNS drugs to treat COVID-19 infection: targeting the sigma-1 receptor. European archives of psychiatry and clinical neuroscience, 1-10 (2021).

81. Vela, J.M. Repurposing sigma-1 receptor ligands for Covid-19 therapy? Frontiers in pharmacology11, 1716 (2020).

82. Abate, C., et al. PB28, the Sigma-1 and Sigma-2 Receptors Modulator With Potent Anti-SARS-CoV-2 Activity: A Review About Its Pharmacological Properties and Structure Affinity Relationships.

Frontiers in pharmacology11(2020). 
83. Yesilkaya, U.H., Balcioglu, Y.H. \& Sahin, S. Reissuing the sigma receptors for SARS-CoV-2. Journal of Clinical Neuroscience80, 72-73 (2020).

84. Salerno, J.A., et al. Inhibition of SARS-CoV-2 infection in human cardiomyocytes by targeting the Sigma-1 receptor disrupts cytoskeleton architecture and contractility. bioRxiv (2021).

85. Hashimoto, K. Review 2:" Inhibition of SARS-CoV-2 infection in human cardiomyocytes by targeting the Sigma-1 receptor disrupts cytoskeleton architecture and contractility". Rapid Reviews COVID-19 (2021).

86. Gordon, D.E., et al. A SARS-CoV-2 protein interaction map reveals targets for drug repurposing. Nature583, 459-468 (2020).

87. Su, H., et al. Discovery of baicalin and baicalein as novel, natural product inhibitors of SARS-CoV-2 $3 \mathrm{CL}$ protease in vitro. BioRxiv (2020).

88. Abdallah, H.M., et al. Repurposing of Some Natural Product Isolates as SARS-COV-2 Main Protease Inhibitors via In Vitro Cell Free and Cell-Based Antiviral Assessments and Molecular Modeling Approaches. Pharmaceuticals14, 213 (2021).

89. Ullrich, S. \& Nitsche, C. The SARS-CoV-2 main protease as drug target. Bioorganic \& Medicinal Chemistry Letters, 127377 (2020).

90. ul Qamar, M.T., Alqahtani, S.M., Alamri, M.A. \& Chen, L.-L. Structural basis of SARS-CoV-2 3CLpro and anti-COVID-19 drug discovery from medicinal plants. Journal of pharmaceutical analysis10, 313-319 (2020).

91. McClain, C.B. \& Vabret, N. SARS-CoV-2: the many pros of targeting PLpro. Signal Transduction and Targeted Therapy5, 1-2 (2020).

92. Klemm, T., et al. Mechanism and inhibition of the papain-like protease, PLpro, of SARS-CoV-2. The EMBO journa/39, e106275 (2020).

93. Rut, W., et al. Activity profiling and crystal structures of inhibitor-bound SARS-CoV-2 papain-like protease: A framework for anti-COVID-19 drug design. Science advances6, eabd4596 (2020).

94. Giordo, R., Zinellu, A., Eid, A.H. \& Pintus, G. Therapeutic potential of resveratrol in COVID-19associated hemostatic disorders. Molecules26, 856 (2021).

95. Rossi, G.A., Sacco, O., Capizzi, A. \& Mastromarino, P. Can Resveratrol-Inhaled Formulations Be Considered Potential Adjunct Treatments for COVID-19? Frontiers in Immunology12, 1591 (2021).

96. Derosa, G., Maffioli, P., D'Angelo, A. \& Di Pierro, F. A role for quercetin in coronavirus disease 2019 (COVID-19). Phytotherapy Research35, 1230-1236 (2021).

97. Yan, Z. \& Caldwell, G.W. Metabolism profiling, and cytochrome P450 inhibition \& induction in drug discovery. Current topics in medicinal chemistry1, 403-425 (2001).

98. De Groot, M.J. Designing better drugs: predicting cytochrome P450 metabolism. Drug discovery today11, 601-606 (2006).

99. Zwaagstra, M.E., et al. Synthesis and Structure- Activity Relationships of Carboxylated Chalcones: A Novel Series of CysLT 1 (LTD4) Receptor Antagonists. Journal of medicinal chemistry40, 1075-1089 
(1997).

100. Bengtson, C.D., et al. An open label trial to assess safety of losartan for treating worsening respiratory illness in COVID-19. Frontiers in medicine8, 152 (2021).

101. Nouri-Vaskeh, M., et al. Comparison of losartan and amlodipine effects on the outcomes of patient with COVID-19 and primary hypertension: A randomised clinical trial. International Journal of Clinical Practice75, e14124 (2021).

102. Puskarich, M.A., et al. A multi-center phase II randomized clinical trial of losartan on symptomatic outpatients with COVID-19. EClinicalMedicine37, 100957 (2021).

103. Geriak, M., et al. Randomized Prospective Open Label Study Shows No Impact on Clinical Outcome of Adding Losartan to Hospitalized COVID-19 Patients with Mild Hypoxemia. Infectious diseases and therapy, 1-8 (2021).

104. Zeinalian, M., Salari-Jazi, A., Jannesari, A. \& Khanahmad, H. A potential protective role of losartan against coronavirus-induced lung damage. Infection Control \& Hospital Epidemiology41, 752-753 (2020).

105. Shen, Z., et al. Potent, Novel SARS-CoV-2 PLpro Inhibitors Block Viral Replication in Monkey and Human Cell Cultures. bioRxiv (2021).

106. Park, J.-Y., et al. Diarylheptanoids from Alnus japonica inhibit papain-like protease of severe acute respiratory syndrome coronavirus. Biological and Pharmaceutical Bulletin, b12-00623 (2012).

107. Seo, W.D., et al. Sulfonamide chalcone as a new class of a-glucosidase inhibitors. Bioorganic \& medicinal chemistry letters 15, 5514-5516 (2005).

108. Williams, S.J. \& Goddard-Borger, E.D. a-glucosidase inhibitors as host-directed antiviral agents with potential for the treatment of COVID-19. Biochemical Society Transactions48, 1287-1295 (2020).

109. Nichols, C., et al. Mining the PDB for Tractable Cases Where X-ray Crystallography Combined with Fragment Screens Can Be Used to Systematically Design Protein-Protein Inhibitors: Two Test Cases Illustrated by IL1 $\beta$-IL1R and p38a-TAB1 Complexes. Journal of Medicinal Chemistry63, 7559-7568 (2020).

110. Ghosh, A.K., et al. Design, synthesis and antiviral efficacy of a series of potent chloropyridyl esterderived SARS-CoV 3CLpro inhibitors. Bioorganic \& medicinal chemistry letters18, 5684-5688 (2008).

111. Welker, A., et al. Structure-Activity Relationships of Benzamides and Isoindolines Designed as SARSCoV Protease Inhibitors Effective against SARS-CoV-2. ChemMedChem16, 340 (2021).

112. Domínguez, J.N., et al. Synthesis and evaluation of new antimalarial phenylurenyl chalcone derivatives. Journal of Medicinal Chemistry48, 3654-3658 (2005).

113. Ratia, K., et al. A noncovalent class of papain-like protease/deubiquitinase inhibitors blocks SARS virus replication. Proceedings of the National Academy of Sciences105, 16119-16124 (2008).

114. Báez-Santos, Y.M., et al. X-ray structural and biological evaluation of a series of potent and highly selective inhibitors of human coronavirus papain-like proteases. Journal of medicinal chemistry57, 2393-2412 (2014). 
115. Wu, C.-Y., et al. Stable benzotriazole esters as mechanism-based inactivators of the severe acute respiratory syndrome 3CL protease. Chemistry \& biology13, 261-268 (2006).

116. Turlington, M., et al. Discovery of $\mathrm{N}$-(benzo $[1,2,3]$ triazol-1-yl)-N-(benzyl) acetamido) phenyl) carboxamides as severe acute respiratory syndrome coronavirus (SARS-CoV) 3CLpro inhibitors: identification of ML300 and noncovalent nanomolar inhibitors with an induced-fit binding. Bioorganic \& medicinal chemistry letters23, 6172-6177 (2013).

117. Hosseini Balef, S.S., Piramoon, M., Hosseinimehr, S.J. \& Irannejad, H. In vitro and in silico evaluation of P-glycoprotein inhibition through 99mTc-methoxyisobutylisonitrile uptake. Chemical biology \& drug design93, 283-289 (2019).

118. Tresanco, M., Tresanco, M., Valiente, P. \& Frías, E. gmx_MMPBSA (Version v1. 4.2). Zenodo. [(accessed on 18 June 2021)](2021).

\section{Tables}

Table 1. The most important host and virus-based targets selected to evaluate the antiviral activity of the 757 chalcone-based compound library, along with PDB codes, chemical structure of their co-crystal ligands, and the authoritative studies proposing these targets as promising strategies for the treatment of viral diseases such as COVID-19. 


\begin{tabular}{|c|c|c|c|c|}
\hline Entry & HBATs & PDB Code & Co-crystallized ligand & HBATs-References \\
\hline 1 & p38 MAPK & $4 \mathrm{EH} 3$ & & $18,26-32$ \\
\hline 2 & Cathepsin L & 5MQY & & $17,33-39$ \\
\hline 3 & $\mathrm{CDK} 1$ & $6 \mathrm{GU} 2$ & & 19,40,44 \\
\hline 4 & $\operatorname{CDK} 2 /$ Cyclin $\mathrm{A}$ & $6 \mathrm{GUB}$ & & 4547 \\
\hline & CDK9/cyclin $\mathrm{T} 1$ & 3BLR & & 48.51 \\
\hline 6 & ERK2 & $3 \mathrm{SA} 0$ & & 52.56 \\
\hline 7 & HDAC2 & $4 \mathrm{LXZ}$ & & 57.61 \\
\hline 8 & DHODH & $5 \mathrm{ZF} 7$ & & 62.68 \\
\hline 9 & CK2 alpha' & $5 \mathrm{M} 4 \mathrm{U}$ & & 69.74 \\
\hline 10 & BRD2 & 4J1P & & $75-77$ \\
\hline 11 & BRD4 & $6 \mathrm{HOV}$ & & $75,77-79$ \\
\hline 12 & $\begin{array}{l}\text { Sigma- } 1 \\
\text { receptor }\end{array}$ & $6 \mathrm{DK} 1$ & & $80-86$ \\
\hline 13 & 3CLpro & $6 \mathrm{M} 2 \mathrm{~N}$ & Baicalein & 87.90 \\
\hline 14 & PLpro & $7 \mathrm{JN} 2$ & Pub & 10,91.93 \\
\hline
\end{tabular}

Table 2. Ranking of the most active hits identified by docking-based virtual screening against various virus and host-based targets. The selected HBATs are named based on their PDB codes. Chalcone-based compounds are named based on their number in our library and encoded as CHA-1 to CHA-757 and the numbers reported in the table body are their docking rank in each molecular target. 


\begin{tabular}{|c|c|c|c|c|c|c|c|c|c|c|c|c|c|c|}
\hline Chalcones & $4 \mathrm{EH} 3$ & $5 \mathrm{MQY}$ & $6 \mathrm{GU} 2$ & 6GUB & 3BLR & $3 \mathrm{SAO}$ & $4 \mathrm{LXZ}$ & 5ZF7 & $5 \mathrm{M} 4 \mathrm{U}$ & $4 \mathrm{~J} 1 \mathrm{P}$ & $6 \mathrm{HOV}$ & 6DK1 & $6 \mathrm{M} 2 \mathrm{~N}$ & 7JN2 \\
\hline CHA-7 & 12 & 72 & 5 & 31 & 19 & 17 & 30 & 31 & 6 & 6 & 6 & 4 & 84 & 5 \\
\hline CHA-10 & 86 & 36 & 64 & 33 & 56 & 24 & 1 & 4 & 156 & 85 & 32 & 36 & 27 & 23 \\
\hline CHA-11 & 231 & 5 & 10 & 1 & 2 & 30 & 19 & 25 & 19 & 13 & 2 & 17 & 36 & 6 \\
\hline CHA-12 & 1 & 2 & 1 & 2 & 3 & 1 & 2 & 1 & 2 & 2 & 3 & 1 & 1 & 1 \\
\hline CHA-15 & 17 & 6 & 6 & 8 & 39 & 24 & 20 & 10 & 11 & 65 & 52 & 8 & 10 & 118 \\
\hline CHA-22 & 153 & 337 & 109 & 263 & 312 & 61 & 566 & 37 & 4 & 191 & 340 & 48 & 161 & 69 \\
\hline CHA-29 & 157 & 220 & 34 & 23 & 25 & 5 & 266 & 145 & 60 & 3 & 43 & 236 & 29 & 99 \\
\hline CHA-30 & 159 & 42 & 53 & 123 & 49 & 33 & 3 & 39 & 209 & 69 & 422 & 52 & 30 & 321 \\
\hline CHA-34 & 4 & 29 & 242 & 96 & 107 & 108 & 14 & 28 & 61 & 50 & 25 & 109 & 52 & 35 \\
\hline CHA-37 & 2 & 4 & 3 & 9 & 7 & 4 & 34 & 9 & 1 & 5 & 1 & 2 & 12 & 3 \\
\hline CHA- 44 & 662 & 16 & 8 & 3 & 1 & 28 & 10 & 73 & 118 & 43 & 5 & 53 & 75 & 4 \\
\hline CHA-60 & 5 & 69 & 358 & 65 & 36 & 49 & 448 & 250 & 123 & 631 & 382 & 44 & 187 & 534 \\
\hline CHA-77 & 13 & 206 & 9 & 4 & 37 & 8 & 136 & 653 & 463 & 63 & 14 & 226 & 193 & 298 \\
\hline CHA-84 & 3 & 650 & 29 & 256 & 753 & 431 & 74 & 756 & 755 & 594 & 716 & 757 & 399 & 755 \\
\hline CHA-86 & 190 & 582 & 381 & 307 & 191 & 214 & 310 & 259 & 79 & 84 & 62 & 62 & 114 & 7 \\
\hline CHA-118 & 674 & 684 & 443 & 529 & 503 & 305 & 654 & 315 & 703 & 439 & 672 & 737 & 405 & 8 \\
\hline CHA-134 & 87 & 384 & 65 & 96 & 107 & 53 & 5 & 556 & 685 & 7 & 23 & 228 & 52 & 44 \\
\hline CHA-166 & 410 & 291 & 30 & 19 & 20 & 26 & 78 & 142 & 54 & 4 & 35 & 159 & 90 & 615 \\
\hline CHA-177 & 31 & 39 & 31 & 42 & 6 & 45 & 9 & 48 & 7 & 8 & 9 & 55 & 91 & 9 \\
\hline CHA-178 & 411 & 553 & 623 & 160 & 199 & 223 & 4 & 67 & 107 & 373 & 133 & 128 & 116 & 240 \\
\hline CHA-206 & 18 & 212 & 228 & 21 & 85 & 3 & 79 & 143 & 206 & 9 & 78 & 78 & 159 & 13 \\
\hline CHA-215 & 357 & 7 & 134 & 11 & 58 & 54 & 59 & 3 & 85 & 115 & 16 & 15 & 611 & 567 \\
\hline CHA-233 & 20 & 27 & 39 & 29 & 130 & 17 & 233 & 20 & 43 & 117 & 101 & 12 & 8 & 401 \\
\hline CHA-234 & 197 & 3 & 32 & 22 & 159 & 19 & 146 & 13 & 22 & 37 & 283 & 5 & 40 & 96 \\
\hline CHA-236 & 7 & 1 & 14 & 13 & 251 & 13 & 114 & 7 & 36 & 118 & 79 & 6 & 3 & 249 \\
\hline CHA-282 & 49 & 122 & 309 & 164 & 73 & 71 & 82 & 108 & 58 & 28 & 226 & 107 & 23 & 2 \\
\hline CHA-297 & 158 & 167 & 15 & 6 & 10 & 14 & 63 & 16 & 59 & 10 & 18 & 37 & 11 & 27 \\
\hline CHA-298 & 50 & 56 & 33 & 45 & 15 & 21 & 24 & 21 & 86 & 38 & 10 & 9 & 68 & 34 \\
\hline CHA-375 & 9 & 86 & 41 & 128 & 8 & 15 & 26 & 5 & 62 & 30 & 11 & 18 & 32 & 19 \\
\hline CHA-378 & 10 & 10 & 24 & 5 & 4 & 16 & 15 & 22 & 63 & 1 & 4 & 28 & 4 & 10 \\
\hline CHA-383 & 373 & 344 & 117 & 393 & 660 & 7 & 513 & 55 & 574 & 515 & 430 & 29 & 6 & 663 \\
\hline CHA-384 & 11 & 88 & 7 & 47 & 63 & 2 & 158 & 2 & 25 & 72 & 7 & 3 & 2 & 37 \\
\hline CHA-392 & 74 & 89 & 55 & 58 & 51 & 34 & 579 & 14 & 261 & 73 & 8 & 14 & 5 & 505 \\
\hline CHA-397 & 24 & 90 & 43 & 48 & 43 & 5 & 126 & 12 & 90 & 102 & 12 & 10 & 7 & 260 \\
\hline CHA-485 & 99 & 288 & 4 & 15 & 34 & 142 & 87 & 388 & 119 & 265 & 300 & 206 & 9 & 59 \\
\hline CHA-509 & 175 & 693 & 18 & 61 & 5 & 48 & 39 & 15 & 176 & 16 & 251 & 59 & 79 & 60 \\
\hline CHA-567 & 81 & 12 & 59 & 63 & 77 & 121 & 170 & 51 & 5 & 20 & 26 & 60 & 44 & 64 \\
\hline CHA-581 & 41 & 443 & 99 & 140 & 44 & 49 & 69 & 30 & 3 & 57 & 27 & 7 & 233 & 64 \\
\hline CHA-734 & 43 & 204 & 2 & 40 & 95 & 96 & 56 & 700 & 462 & 12 & 13 & 443 & 111 & 384 \\
\hline
\end{tabular}

Bolded ligands are the best hits selected based on their rank number for further evaluations.

Most active ligands selective towards PL pro target. Lighter colors indicate lower magnitude of affinity.

Most active ligands towards 3CLpro and PLpro targets. Lighter colors indicate lower magnitude of affinity. $\square$ Most active ligands selective towards 3CLpro target. Lighter colors indicate lower magnitude of affinity. 
Table 3

Binding free energy values and energy components of the compounds in the 3 CLpro active site calculated by MM/PB(GB)SA in $\mathrm{kcal} / \mathrm{mol}$.

\begin{tabular}{|llllllllll|}
\hline \multicolumn{1}{c}{ GB } & \multicolumn{7}{c|}{$\mathrm{PB}$} \\
\hline & $\Delta \mathrm{G}_{\mathrm{b}}$ & $\Delta \mathrm{G}_{\text {Total }}$ & $\Delta \mathrm{G}_{\text {solv }}$ & $\Delta \mathrm{G}_{\text {gas }}$ & $\Delta \mathrm{G}_{\mathrm{b}}$ & $\Delta \mathrm{G}_{\text {Total }}$ & $\Delta \mathrm{G}_{\text {solv }}$ & $\Delta \mathrm{G}_{\text {gas }}$ & $\mathrm{IE}$ \\
\hline Baicalein & -30.93 & -31.77 & 7.19 & -38.97 & -26.40 & -27.24 & 11.73 & -38.97 & 0.84 \\
\hline CHA-12 & -38.57 & -38.0 & 24.04 & -62.05 & -32.36 & -31.8 & 30.25 & -62.05 & -0.56 \\
\hline CHA-384 & -33.37 & -37.28 & 15.77 & -53.05 & -25.13 & -29.04 & 24.01 & -53.05 & 3.91 \\
\hline CHA-37 & -31.09 & -33.96 & 11.28 & -45.24 & -20.66 & -23.53 & 21.70 & -45.24 & 2.87 \\
\hline CHA-297 & -20.74 & -21.60 & 26.31 & -47.91 & -17.83 & -18.69 & 22.29 & -47.91 & 0.85 \\
\hline CHA-378 & -14.24 & -18.75 & 24.10 & -42.85 & -13.99 & -18.5 & 24.35 & -42.85 & 4.51 \\
\hline Hirsutenone & -24.12 & -29.04 & 9.02 & -38.07 & -18.69 & -23.61 & 14.45 & -38.07 & 4.92 \\
\hline A & -11.96 & -12.41 & 26.89 & -39.30 & -16.49 & -16.95 & 22.35 & -39.30 & 0.46 \\
\hline B & -20.36 & -21.76 & 8.15 & -29.91 & -12.71 & -14.1 & 15.8 & -29.91 & 1.40 \\
\hline
\end{tabular}

Table 4

Binding free energy values and energy components of the compounds in the PLpro active site calculated by $\mathrm{MM} / \mathrm{PB}(\mathrm{GB}) \mathrm{SA}$ in $\mathrm{kcal} / \mathrm{mol}$.

\begin{tabular}{|llllllllll|}
\hline \multicolumn{1}{c}{ GB } & \multicolumn{7}{c|}{$\mathrm{PB}$} \\
& $\Delta \mathrm{G}_{\mathrm{b}}$ & $\Delta \mathrm{G}_{\mathrm{T}}$ & $\Delta \mathrm{G}_{\text {solv }}$ & $\Delta \mathrm{G}_{\text {gas }}$ & $\Delta \mathrm{G}_{\mathrm{b}}$ & $\Delta \mathrm{G}_{\mathrm{T}}$ & $\Delta \mathrm{G}_{\text {solv }}$ & $\Delta \mathrm{G}_{\text {gas }}$ & $\mathrm{IE}$ \\
\hline $\begin{array}{l}\text { Co-crystal } \\
\text { ligand }\end{array}$ & -18.37 & -19.02 & 9.30 & -28.32 & -14.22 & -14.87 & 13.45 & -28.32 & 0.65 \\
\hline GRL0617 & -31.33 & -34.11 & 19.82 & -53.94 & -23.65 & -26.42 & 27.51 & -53.94 & 2.78 \\
CHA-12 & -32.86 & -34.81 & 16.75 & -51.56 & -27.85 & -29.80 & 21.76 & -51.56 & 1.95 \\
CHA-37 & -21.20 & -21.95 & 16.96 & -38.91 & -19.20 & -19.95 & 18.96 & -38.91 & 0.75 \\
\hline CHA-378 & -11.01 & -18.49 & 18.49 & -36.99 & -9.34 & -16.82 & 20.16 & -36.99 & 7.48 \\
\hline Hirsutenone & -22.67 & -32.29 & 14.82 & -47.12 & -16.52 & -26.14 & 20.98 & -47.12 & 9.62 \\
\hline
\end{tabular}

Table 5. Some important druglikeness, ADME, and toxicity parameters of the best identified CHA-12, CHA37, CHA-378, CHA-384, and standards quercetin and resveratrol predicted by admetSAR, preADME, and swissADME online servers. 


\begin{tabular}{lcccccc}
\hline Parameters & CHA-12 & CHA-37 & CHA-378 & CHA-384 & Quercetin & Resveratrol \\
\hline Formula & $\mathrm{C}_{26} \mathrm{H}_{19} \mathrm{~N}_{5} \mathrm{O}_{3}$ & $\mathrm{C}_{21} \mathrm{H}_{15} \mathrm{~N}_{3} \mathrm{O}$ & $\mathrm{C} 22 \mathrm{H} 19 \mathrm{NO} 5 \mathrm{~S}$ & $\mathrm{C}_{23} \mathrm{H}_{17} \mathrm{ClN}_{2} \mathrm{O}_{4}$ & $\mathrm{C}_{13} \mathrm{H}_{10} \mathrm{O}_{7}$ & $\mathrm{C}_{14} \mathrm{H}_{12} \mathrm{O}_{3}$ \\
Molecular weight $(\mathrm{g} / \mathrm{mol})$ & 449.46 & 325.36 & 409.45 & 420.85 & 302.24 & 228.24 \\
Lipinski rule of Five & Suitable & Suitable & Suitable & Suitable & Suitable & Suitable \\
Rule of Five Violationsa & 0 & 0 & 0 & 0 & 0 & 0 \\
BBB permeant & No & Yes & No & No & No & Yes \\
GI absorption & High & High & High & High & High & High \\
P-glycoprotein Inhibitor & No & Inhibitor & No & No & No & No \\
Acute Oral Toxicity & III & III & III & III & II & III \\
Carcinogens & No & No & No & No & No & No \\
\hline
\end{tabular}

a: Violations of Lipinski rule of $5(\log \mathrm{P}<5, \mathrm{MW}<500, \mathrm{nHBA}<10$, and $\mathrm{nHBD}<5)$.

Table 6. Predicted inhibitory effects of CHA-12, CHA-37, CHA-378, CHA-384, resveratrol, and quercetin on cytochrome P450 enzymes (predicted by preADME online server).

\begin{tabular}{lcccccc}
\hline Compounds & $\begin{array}{c}\text { CYP2C19 } \\
\text { inhibition }\end{array}$ & $\begin{array}{c}\text { CYP2C9 } \\
\text { inhibition }\end{array}$ & $\begin{array}{c}\text { CYP2D6 } \\
\text { inhibition }\end{array}$ & $\begin{array}{c}\text { CYP2D6 } \\
\text { Substrate }\end{array}$ & $\begin{array}{c}\text { CYP3A4 } \\
\text { inhibition }\end{array}$ & $\begin{array}{c}\text { CYP3A4 } \\
\text { Substrate }\end{array}$ \\
\hline CHA-12 & Non & Inhibitor & Non & Non & Non & Weakly \\
CHA-37 & Non & Inhibitor & Non & Non & Non & Weakly \\
CHA-378 & Inhibitor & Inhibitor & Non & Non & Inhibitor & Non \\
CHA-384 & Non & Inhibitor & Non & Non & Non & Non \\
Resveratrol & Inhibitor & Inhibitor & Non & Non & Inhibitor & Non \\
Quercetin & Inhibitor & Inhibitor & Non & Non & Inhibitor & Non \\
\hline
\end{tabular}

Table 7. Important interactions of most active CHA-12, CHA-378, CHA-384, and baicalein as standard in the SARS-CoV-2 3CLpro active site 


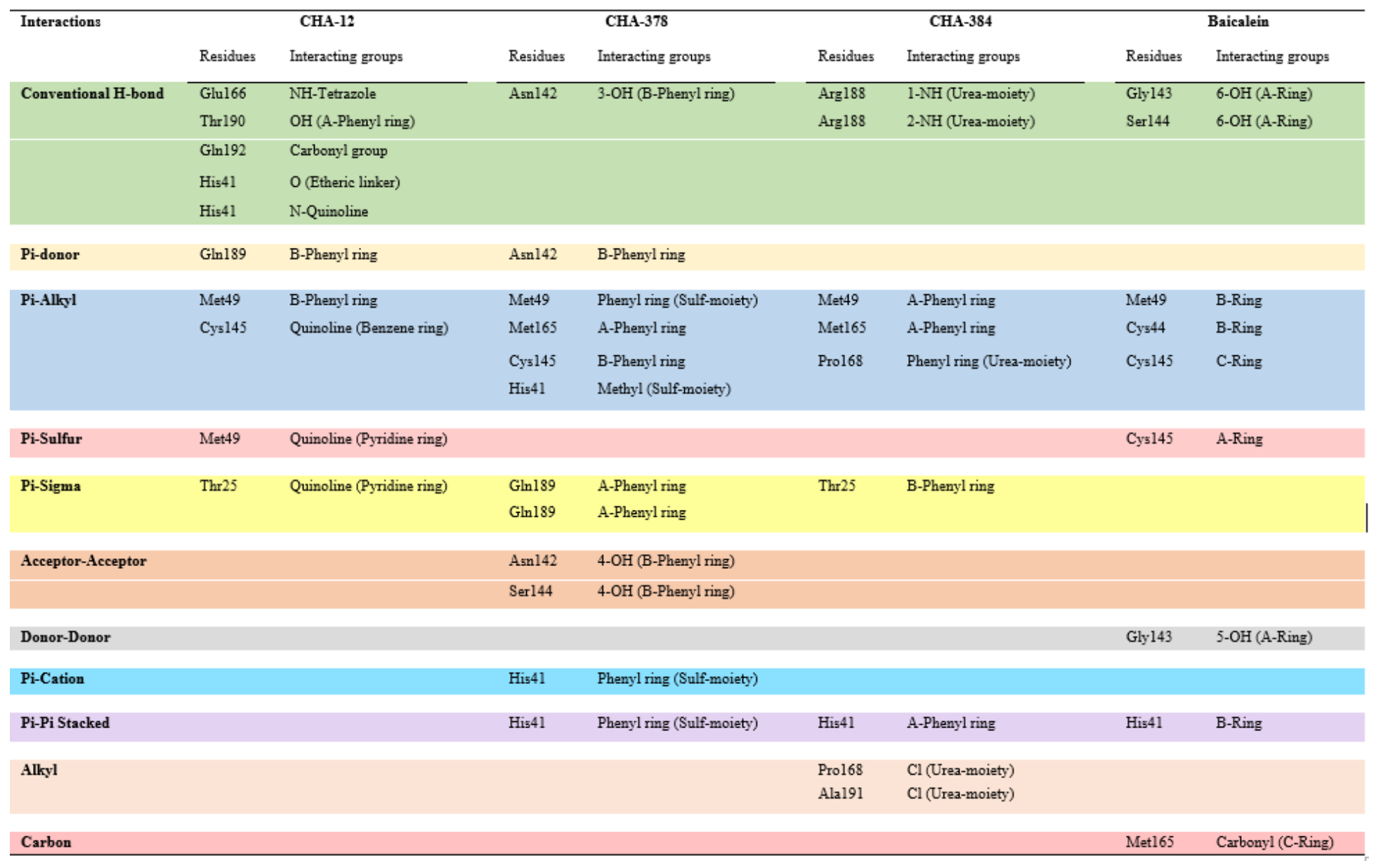

Table 8. Important interactions of the most active CHA-12, CHA-37, CHA-378 and standard GRL0617 in the active site of SARS-CoV-2 PLpro. 


\begin{tabular}{|c|c|c|c|c|c|c|c|c|}
\hline \multirow[t]{2}{*}{ Interactions } & \multicolumn{2}{|r|}{ CHA-12 } & \multicolumn{2}{|r|}{ CHA-37 } & \multicolumn{2}{|r|}{ CHA-384 } & \multicolumn{2}{|r|}{ GRL0617 } \\
\hline & Residues & Interacting groups & Residues & Interacting groups & Residues & Interacting groups & Residues & Interacting groups \\
\hline \multirow[t]{3}{*}{ Conventional H-bond } & Lys 157 & N1 Tetrazole & & & Tyr264 & Carbonyl group & $\mathrm{G} \ln 269$ & Carbonyl group \\
\hline & $\mathrm{G} \ln 269$ & N4 Tetrazole & & & Glul67 & $\mathrm{NH}$ (Sulf-moiety) & Aspl64 & NH (Amide) \\
\hline & Tyr264 & OH (A-Phenyl ring) & & & & & Glyl63 & NH2 (A-Phenyl ring) \\
\hline Pi-donor & $G \ln 269$ & Tetrazole ring & & & & & & \\
\hline \multirow[t]{4}{*}{ Pi-Alkyl } & Pro 248 & B-Phenyl ring & Pro 248 & B-Phenyl ring & Pro 248 & B-Phenyl ring & Tyr268 & $\mathrm{CH}_{3}$ (A-Phenyl ring) \\
\hline & & & Pro 248 & Triazole (Benzotriazole) & Leul62 & A-Phenyl ring & Pro 247 & Naphthyl (Closer ring) \\
\hline & & & Pro 248 & Benzene (Benzotriazole) & & & Pro 248 & Naphthyl (Closer ring) \\
\hline & & & Pro 247 & Benzene (Benzotriazole) & & & Pro 248 & Naphthyl (Farther ring) \\
\hline \multirow[t]{2}{*}{ Amide-Pi Stacked } & Gly163 & A-Phenyl ring & Glyl63 & A-Phenyl ring & Glyl63 & A-Phenyl ring & & \\
\hline & & & $A=267$ & Triazole (Benzotriazole) & & & & \\
\hline Pi-Sigma & & & & & $\mathrm{G} \ln 269$ & A-Phenyl ring & Aspl64 & A-Phenyl ring \\
\hline Acceptor-Acceptor & Tyr273 & Carbonyl group & & & & & & \\
\hline Pi-Cation & & & Lys 157 & A-Phenyl ring & & & & \\
\hline \multirow[t]{2}{*}{ Pi-Anion } & Glul67 & Tetrazole ring & & & Aspl64 & B-Phenyl ring & Aspl64 & A-Phenyl ring \\
\hline & & & & & Glul67 & Phenyl ring (Sulf-moiety) & & \\
\hline \multirow[t]{2}{*}{ Pi-Pi Stacked } & Tyr268 & Quinoline (Pyridine ring) & Tyr264 & B-Phenyl ring & & & & \\
\hline & Tyr268 & Quinoline (Benzene ring) & & & & & & \\
\hline \multirow[t]{2}{*}{ Pi-Pi T-Shaped } & Tyr268 & B-Phenyl ring & Tyr268 & Benzene (Benzotriazole) & Tyr264 & B-Phenyl ring & Tyr268 & Naphthyl (Closer ring) \\
\hline & & & & & & & Tyr268 & Naphthyl (Farther ring) \\
\hline
\end{tabular}

Table 9. The list of host-based and virus-based targets along with their associated PDB codes as well as the essential parameters for the docking protocol used in this study. 


\begin{tabular}{lllllll} 
Entry & Target & PDB Code & $\mathbf{X}$ & $\mathbf{Y}$ & $\mathbf{Z}$ & Grid box \\
\hline $\mathbf{1}$ & p38 MAPK & 4EH3 & -2.668 & 0.007 & -20836 & $20,20,20$ \\
\hline $\mathbf{2}$ & Cathepsin L & 5MQY & 54.36 & 48.381 & 17.63 & $20,20,20$ \\
\hline $\mathbf{3}$ & CDK1 & 6GU2 & 329.218 & 212.541 & 192.325 & $20,20,20$ \\
\hline $\mathbf{4}$ & CDK2/CyclinA & 6GUB & -8.411 & -21.607 & 22.226 & $20,20,20$ \\
\hline $\mathbf{5}$ & CDK9/cyclinT1 & 3BLR & 52.813 & -16.066 & -12.998 & $20,20,20$ \\
\hline $\mathbf{6}$ & ERK2 & 3SA0 & -12.826 & 10.831 & 40.627 & $26,22,20$ \\
\hline $\mathbf{7}$ & HDAC2 & 4LXZ & 25.00 & -17.005 & -0.024 & $20,20,20$ \\
\hline $\mathbf{8}$ & DHODH & 5ZF7 & -33.443 & 14.428 & -21.466 & $20,22.22$ \\
\hline $\mathbf{9}$ & CK2 alpha' & 5M4U & 10.536 & -19.326 & -8.976 & $20,20,20$ \\
\hline $\mathbf{1 0}$ & RBD2 & 4J1P & 10.105 & 20.989 & -6.705 & $20,20,26$ \\
\hline $\mathbf{1 1}$ & RBD4 & 6HOV & 10.887 & 5.487 & -0.524 & $20,20,20$ \\
\hline $\mathbf{1 2}$ & Sigma-1 receptor & 6DK1 & -21.013 & 6.051 & -18.412 & $20,20,22$ \\
\hline $\mathbf{1 3}$ & SARS-CoV-2 3CLPro & 6M2N & -32.488 & -63.85 & 40.962 & $22,20,20$ \\
\hline $\mathbf{1 4}$ & SARS-CoV-2 PLPro & 7JN2 & 51.608 & 30.592 & 0.79 & $20,20,20$ \\
\hline
\end{tabular}

Figures 
<smiles>C=C(C)C(Cc1c(OC)ccc(C(=O)/C=C/c2ccc(O)cc2)c1O)OO</smiles>

Xanthoangelol E

3CLPro inhibition, $\mathrm{IC}_{50}=11.4 \pm 1.4 \mu \mathrm{M}$

PLPro inhibition, $\quad \mathrm{IC}_{50}=1.2 \pm 0.4 \mu \mathrm{M}$<smiles>C=C(C)C(O)CC/C(C)=C/Cc1c(O)ccc(C(=O)/C=C/c2ccc(O)cc2)c1O</smiles>

Xanthoangelol B

3CLPro inhibition, $\mathrm{IC}_{50}=22.2 \pm 6.5 \mu \mathrm{M}$

PLPro inhibition, $\quad I C_{50}=11.7 \pm 0.3 \mu \mathrm{M}$<smiles>C=C(C)C(O)Cc1c(OC)ccc(C(=O)/C=C/c2ccc(O)cc2)c1O</smiles>

Xanthoangelol D

3CLPro inhibition, $I_{50}=26.6 \pm 5.2 \mu \mathrm{M}$

PLPro inhibition, $\quad \mathrm{IC}_{50}=19.3 \pm 1.8 \mu \mathrm{M}$

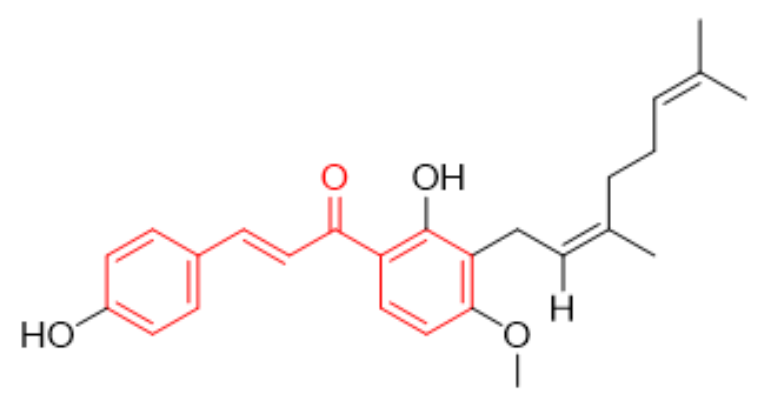

Xanthoangelol F

3CLPro inhibition, $\mathrm{IC}_{50}=34.1 \pm 4.8 \mu \mathrm{M}$

PLPro inhibition, $\quad \mathrm{IC}_{50}=5.6 \pm 0.5 \mu \mathrm{M}$

Figure 1

Chemical structures of some natural-based chalcones having anti-SARS CoV activity. 


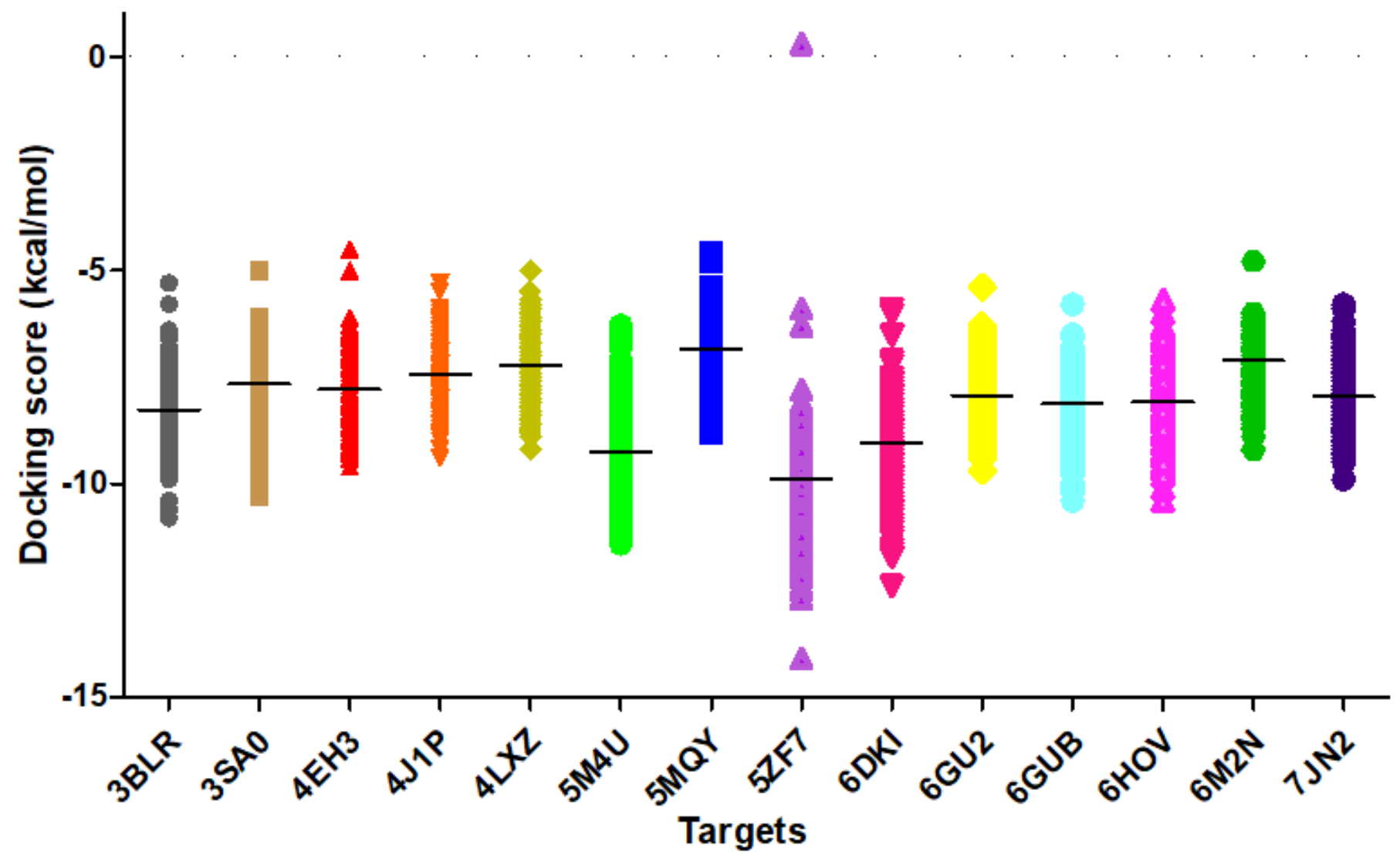

Figure 2

Docking score $(\mathrm{kcal} / \mathrm{mol})$ distribution of the 757 chalcone-based compound library over the 12 hostbased molecular targets consisted of CDK9/cyclinT1 (3BLR), ERK2 (3SA0), p38 MAPK (4EH3), RBD2 (4J1P), HDAC2 (4LXZ), CK2 alpha' (5M4U), Cathepsin L (5MQY), DHODH (5ZF7), Sigma-1 receptor (6DK1), CDK1 (6GU2), CDK2/CyclinA (6GUB), , RBD4 (6HOV), and the two viral targets 3CLpro (6M2N), and PLpro (7JN2). The intersecting line in each cluster reperesents the average docking score in each target. 
<smiles>O=C(/C=C/CCc1ccc(O)c(O)c1)CCc1ccc(O)c(O)c1</smiles>

Hirsutenone

SARS-CoV PLpro inhibition, $\mathrm{IC}_{50}: 4.1 \mu \mathrm{M}$

SARS-CoV 3CLpro inhibition, $I \mathrm{IC}_{50}: 36.2 \mu \mathrm{M}$<smiles>Nc1ccc(S(=O)(=O)Nc2ccccn2)cc1</smiles>

A

SARS-CoV-2 3CLpro-inhibitor (PDB Code: 5RF8)

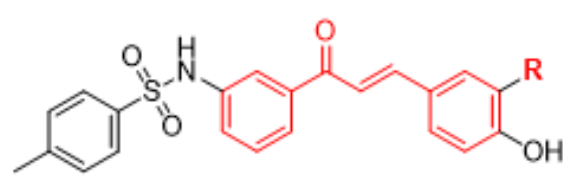

$\mathrm{R}=\mathrm{OH}, \mathrm{CHA}-378$

$\mathrm{R}=\mathrm{H}, \quad$ CHA-297<smiles>O=c1cc(-c2ccccc2)oc2cc(O)c(O)c(O)c12</smiles>

Baicalein

SARS-CoV-2 3CLpro inhibition,

$\mathrm{IC}_{50}: 0.39 \pm 0.11 \mu \mathrm{M}$<smiles>Cc1ccc(N)cc1C(=O)N[C@@H](C)c1cccc2ccccc12</smiles>

GRL0617

SARS-CoV PLpro Inhibition, $\mathrm{IC}_{50}=0.6 \mu \mathrm{M}$ SARS-CoV-2 PLpro Inhibition, $\mathrm{IC}_{50}=1.6-2.2 \mu \mathrm{M}$<smiles>O=C(Nc1ccccc1)Nc1cccnc1</smiles>

B

SARS-CoV-2 3CLpro-inhibitor (PDB Code: 5R83)<smiles>O=C(/C=C/c1ccc(OCc2ccc3ccccc3n2)cc1)c1cc(-c2nn[nH]n2)ccc1O</smiles>

CHA-12 (VUF 4819)<smiles>O=C(Nc1ccc(Cl)cc1)Nc1ccc(C(=O)/C=C/c2ccc3c(c2)OCO3)cc1</smiles>

CHA-384

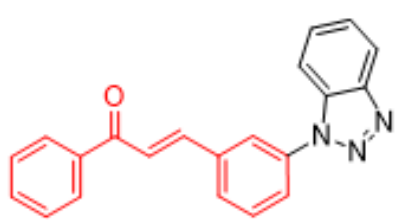

CHA-37

\section{Figure 3}

Chemical structure of some of the famous SARS-CoV-2 inhibitors hirsutenone, baicalein, GRL0617 and their experimentally reported potencies; and also newly reported SARS-CoV-2 3CLpro fragment inhibitors A and B. The most active compounds CHA-12, CHA-37, CHA-297, CHA-378, and CHA-384 were selected as the promising chalcone-based structures for the inhibition of SARS-CoV-2, identified by our preliminary in silico screening protocol. 


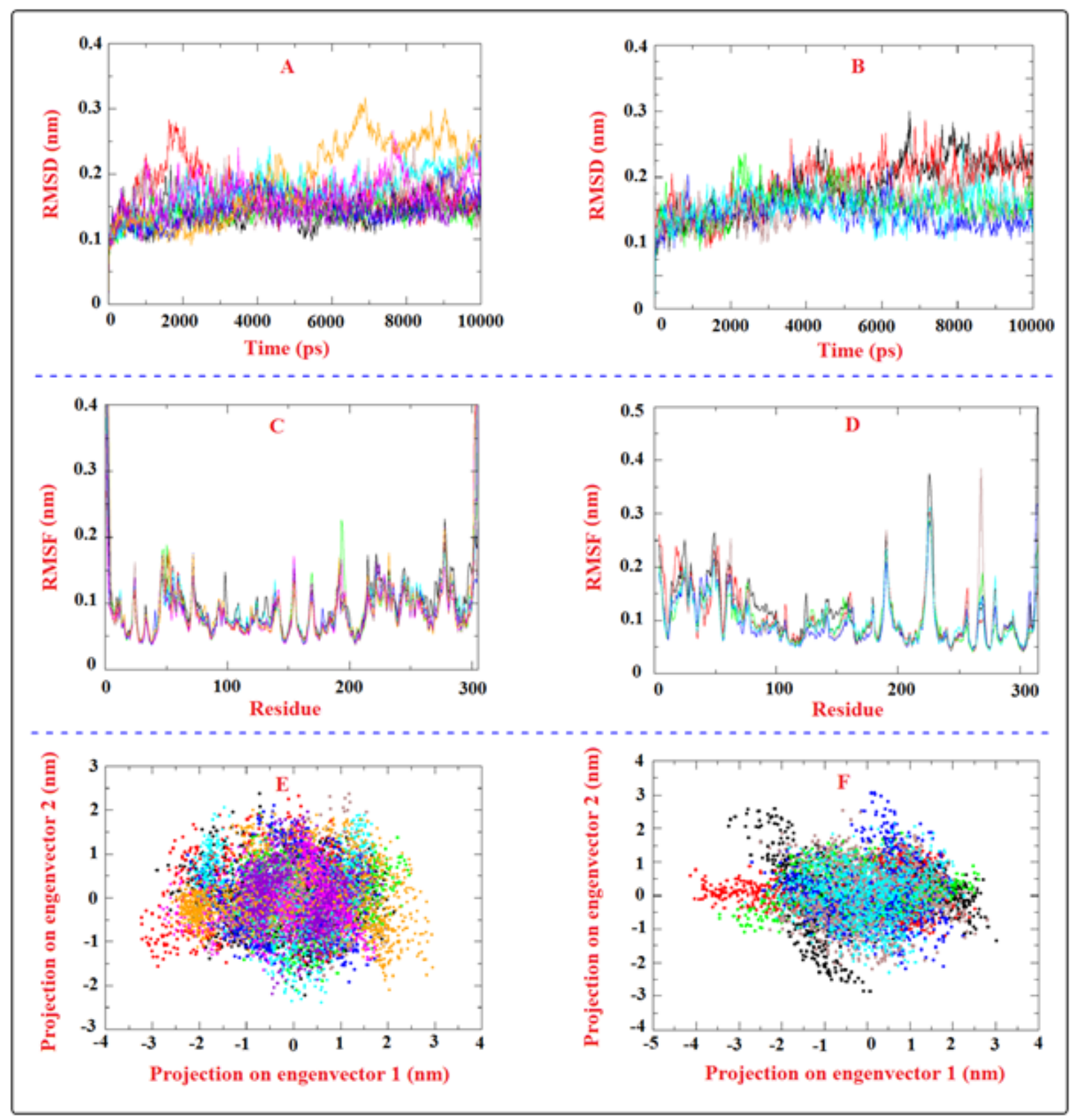

Figure 4

A) RMSD plot for Ca variations during the MD simulation time $10 \mathrm{~ns}$ for the 3CLpro and PLpro complexes. B) RMSF plot for Ca variations during the MD simulation time $10 \mathrm{~ns}$ for the 3 CLpro and PLpro complexes. C) 2D projection of the first and second eigenvectors for the 3CLpro and PLpro complexes. For the 3CLpro complexes: black: baicalin, red: CHA-12, green: CHA-384, blue: CHA-37, brown: CHA-297, cyan: CHA-378, magenta: hirsutenone, violet: compound $A$ and orange: compound $B$. For the PLpro complexes: Black: co-crystal ligand, red: GRL0617, green: CHA-12, blue: CHA-37, brown: CHA-378 and cyan: hirsutanone. 


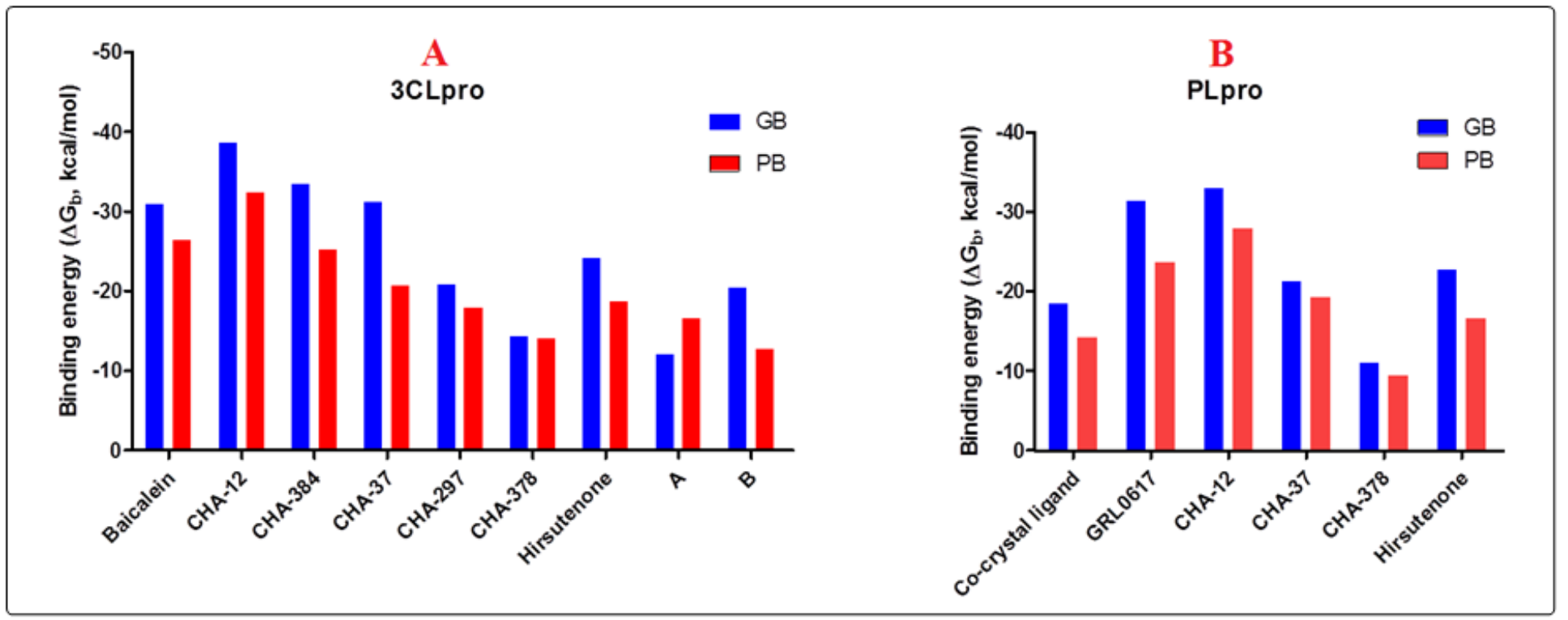

Figure 5

Binding free energy $(\triangle \mathrm{Gb})$ of compounds calculated by the GB (blue) and PB (red) methods in the active sites of the 3CLpro (A) and PLpro (B). 


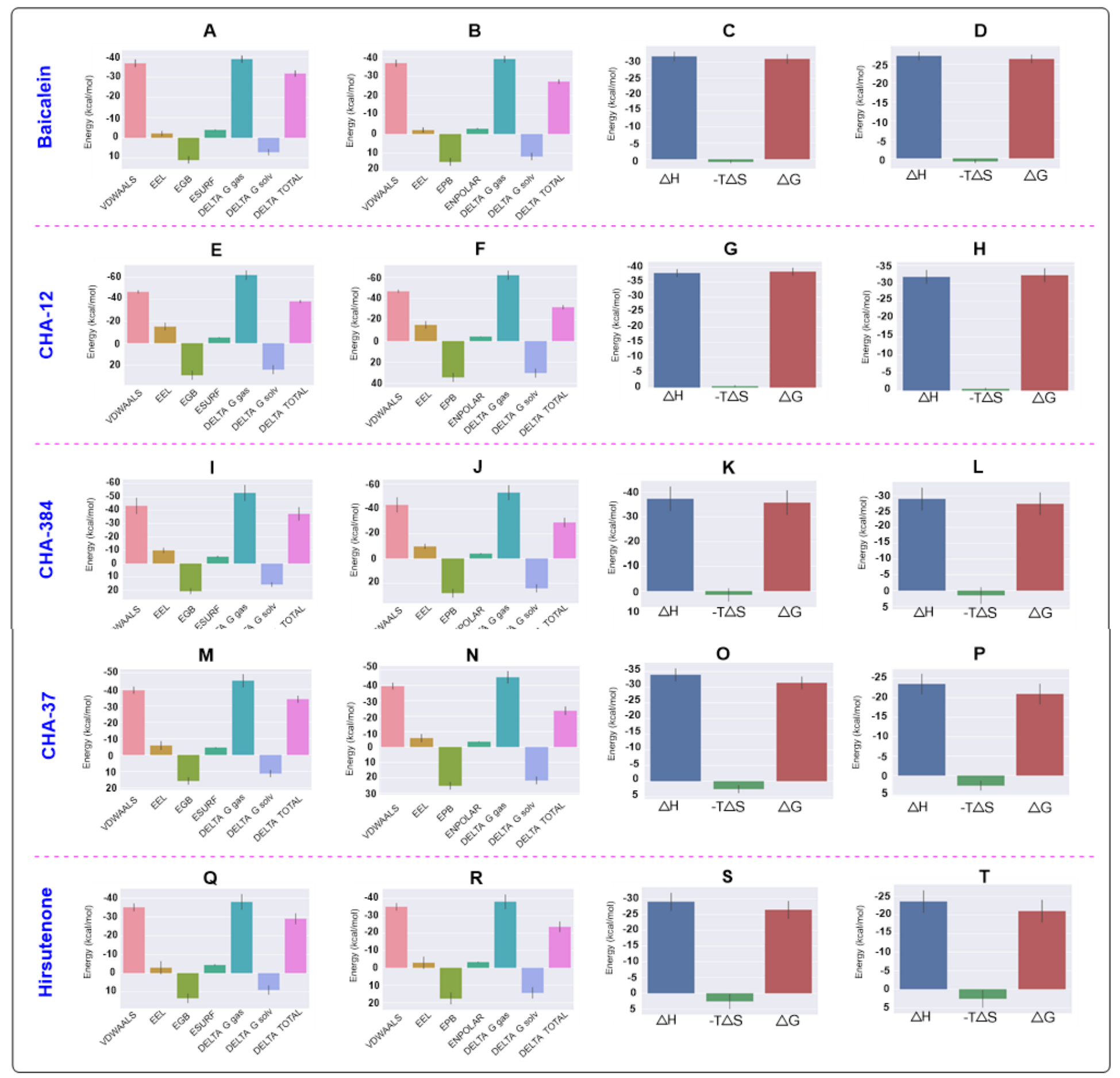

\section{Figure 6}

Energy components of the compounds calculated by the GB and PB in the 3CLPro. Energy components of baicalein in $\mathrm{GB}(\mathrm{A})$ and $\mathrm{PB}(\mathrm{B})$ analysis and its binding free energy $(\triangle \mathrm{Gb})$ decomposed to the enthalpy $(\Delta \mathrm{H})$ and entropy $(-\mathrm{T} \Delta \mathrm{S})$ terms in the $\mathrm{GB}(\mathrm{C})$ and $\mathrm{PB}(\mathrm{D})$ calculations. Correspondingly, energy components and binding free energy $(\triangle \mathrm{Gb})$ terms for $\mathrm{CHA}-12$ in the $\mathrm{GB}$ and $\mathrm{PB}$ calculations are respectively illustrated in $\mathrm{E}, \mathrm{F}, \mathrm{G}$ and $\mathrm{H}$. Energy components and binding free energy $(\Delta \mathrm{Gb})$ terms for $\mathrm{CHA}$ 384 in the GB and PB calculations are respectively illustrated in I, J, K and L. Energy components and binding free energy $(\triangle \mathrm{Gb})$ terms for CHA-37 in the GB and PB calculations are respectively illustrated in 
$\mathrm{M}, \mathrm{N}, \mathrm{O}$ and $\mathrm{P}$. Energy components and binding free energy $(\triangle \mathrm{Gb})$ terms for hirsutenone in the $\mathrm{GB}$ and $\mathrm{PB}$ calculations are respectively illustrated in $\mathrm{Q}, \mathrm{R}, \mathrm{S}$ and $\mathrm{T}$. VDWAALS: van der Waals energy, EEL: electrostatic energy, EGB: Polar solvation energy calculated by the GB, ESURF: Non-polar solvation energy calculated by the GB, EPB: Polar solvation energy calculated by the PB, ENPOLAR: Non-polar solvation energy calculated by the PB.

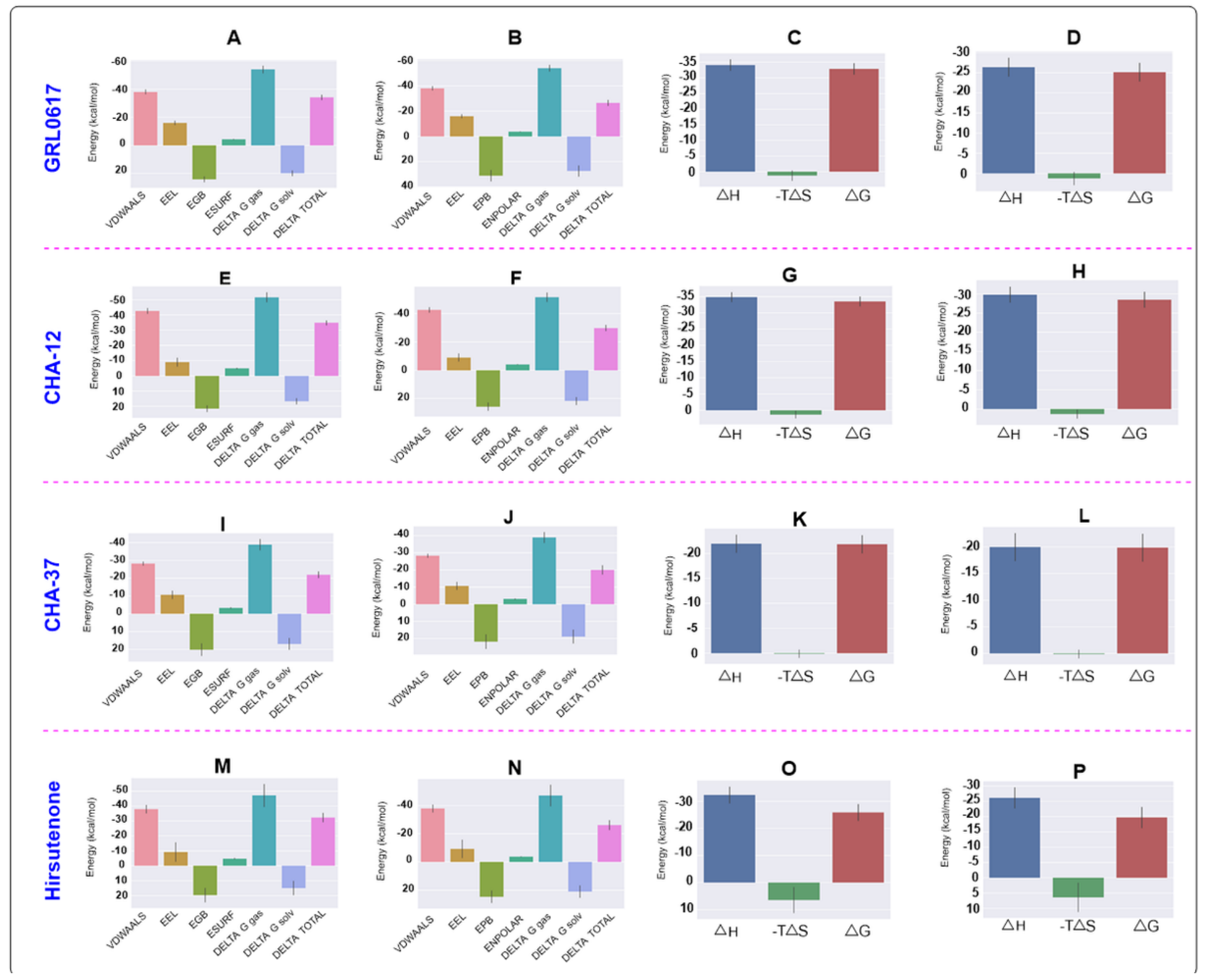

\section{Figure 7}

Energy components of the compounds calculated by the GB and PB in the PLpro. Energy components of GRL0617 in the GB (A) and PB (B) analysis and its binding free energy $(\triangle \mathrm{Gb})$ decomposed to the enthalpy $(\Delta \mathrm{H})$ and entropy $(-\mathrm{T} \Delta \mathrm{S})$ terms in the GB (C) and PB (D) calculations. Correspondingly, energy components and binding free energy $(\triangle \mathrm{Gb})$ terms for $\mathrm{CHA}-12$ in the $\mathrm{GB}$ and the $\mathrm{PB}$ calculations are respectively illustrated in $\mathrm{E}, \mathrm{F}, \mathrm{G}$ and $\mathrm{H}$. Energy components and binding free energy $(\Delta \mathrm{Gb})$ terms for $\mathrm{CHA}$ 37 in the GB and PB calculations are respectively illustrated in I, J, K and L. Energy components and binding free energy $(\Delta \mathrm{Gb})$ terms for hirsutenone in the GB and PB calculations are respectively illustrated 
in $\mathrm{M}, \mathrm{N}, \mathrm{O}$ and P. VDWAALS: van der Waals energy, EEL: electrostatic energy, EGB: Polar solvation energy calculated by the GB, ESURF: Non-polar solvation energy calculated by the GB, EPB: Polar solvation energy calculated by the PB, ENPOLAR: Non-polar solvation energy calculated by the PB.

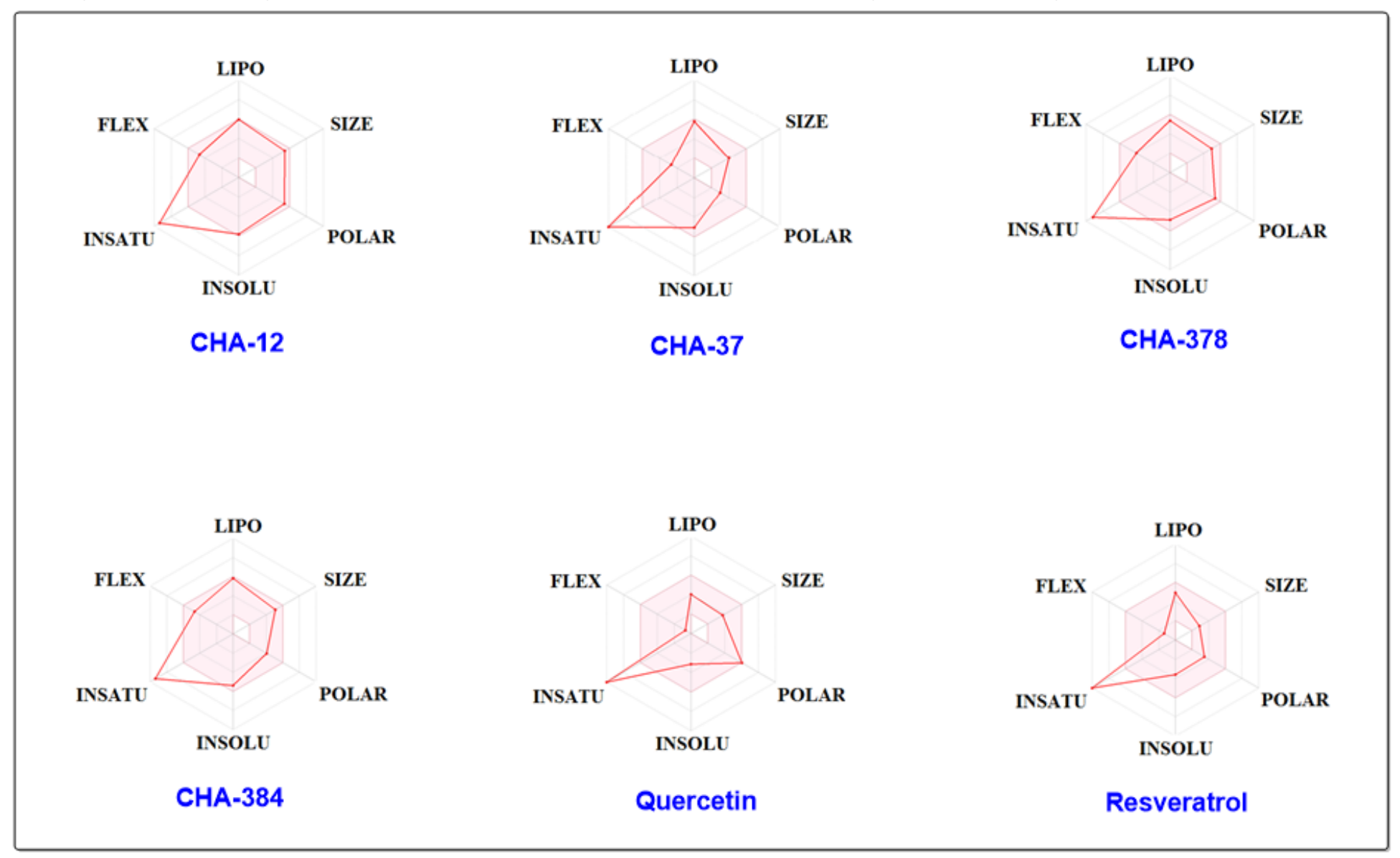

\section{Figure 8}

Radar map of the physicochemical properties for oral bioavailability of CHA-12, CHA-37, CHA-378, CHA384 , and standards quercetin and resveratrol predicted by SwissADME online server (colored zone is the suitable physicochemical space for oral bioavailability). LIPO (Lipophilicity): $-0.7<$ XLOGP3 < +5.0; SIZE: $150 \mathrm{~g} / \mathrm{mol}<\mathrm{mw}<500 \mathrm{~g} / \mathrm{mol}$; POLAR (polarity): 20 Å2 < TPSA < 130 Å2; INSOLU (insolubility): $0<$ Log S (ESOL) < 6; INSATU (insaturation): $0.25<$ Fraction Csp3 < 1; FLEX (Flexibility): $0<$ Numb. rotatable bonds $<9$. 

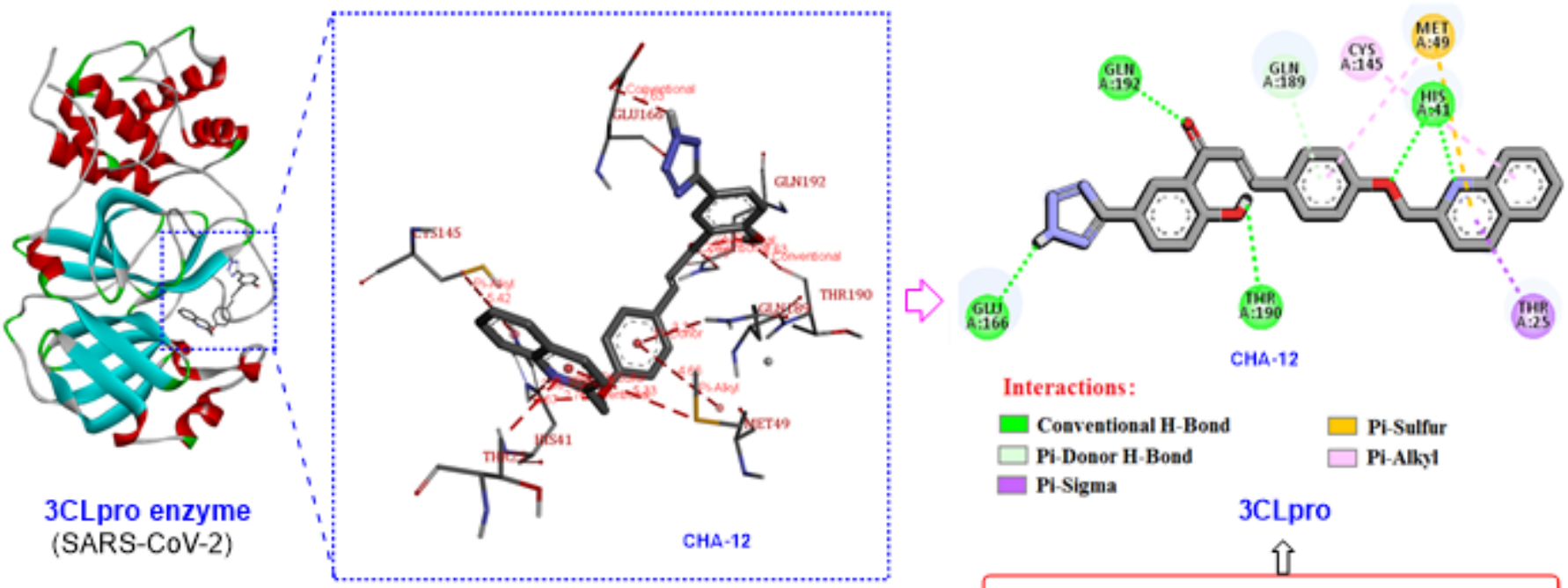

Interactions:

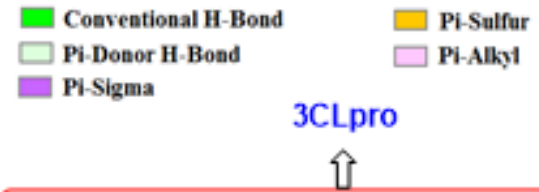

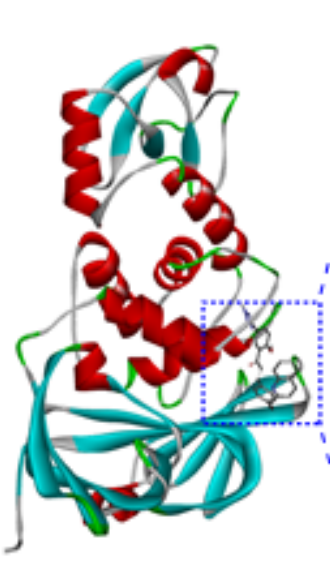

PLpro enzyme (SARS-CoV-2)

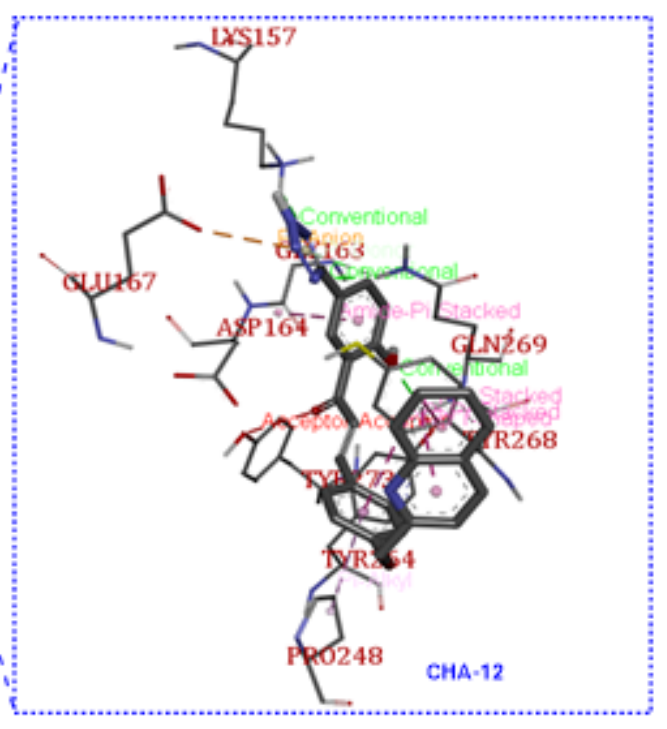

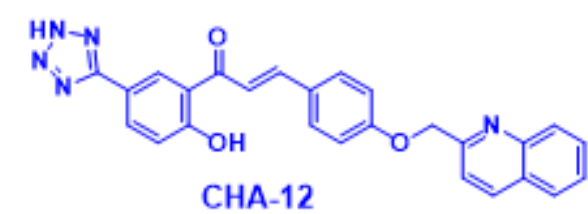

गु

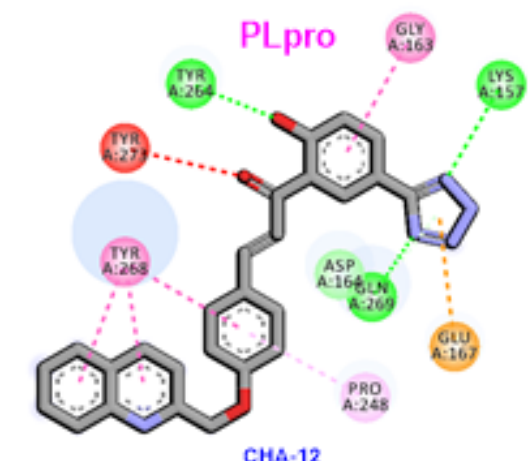

Intractions:

$\square$ Vas der Wask $\square$ Pi-Pi Stacked

- Conventional H-Bond

Di-Pi T-Shaped

- Unfavorable Accepter-Acceptor

[- Pi-Anion

$\square$ Pi-Donor H-bend

$\square$ Pi-Alkyl

\section{Figure 9}

2D and 3D intractions of CHA-12 in the active site of the SARS-CoV-2 3CLpro and PLpro enzymes.

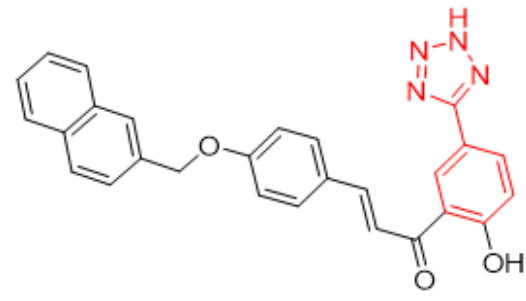

CHA-12

(PubChem CID: 135430849)

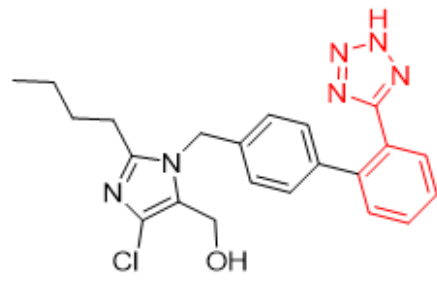

Losartan

(In clinical trials for COVID-19)

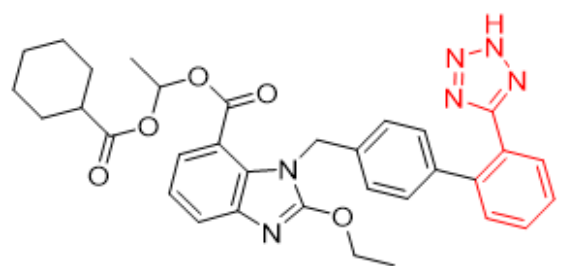

Candesartan cilexetil (Weak SARS-CoV-2 PLpro Inhibitor, $\left.\mathrm{IC}_{50}=>10 \mu \mathrm{M}\right)$ 
Structural similarity of $\mathrm{CHA}-12$, losartan, and candesartan cilexetil due to the existence of a phenyltetrazole moiety.

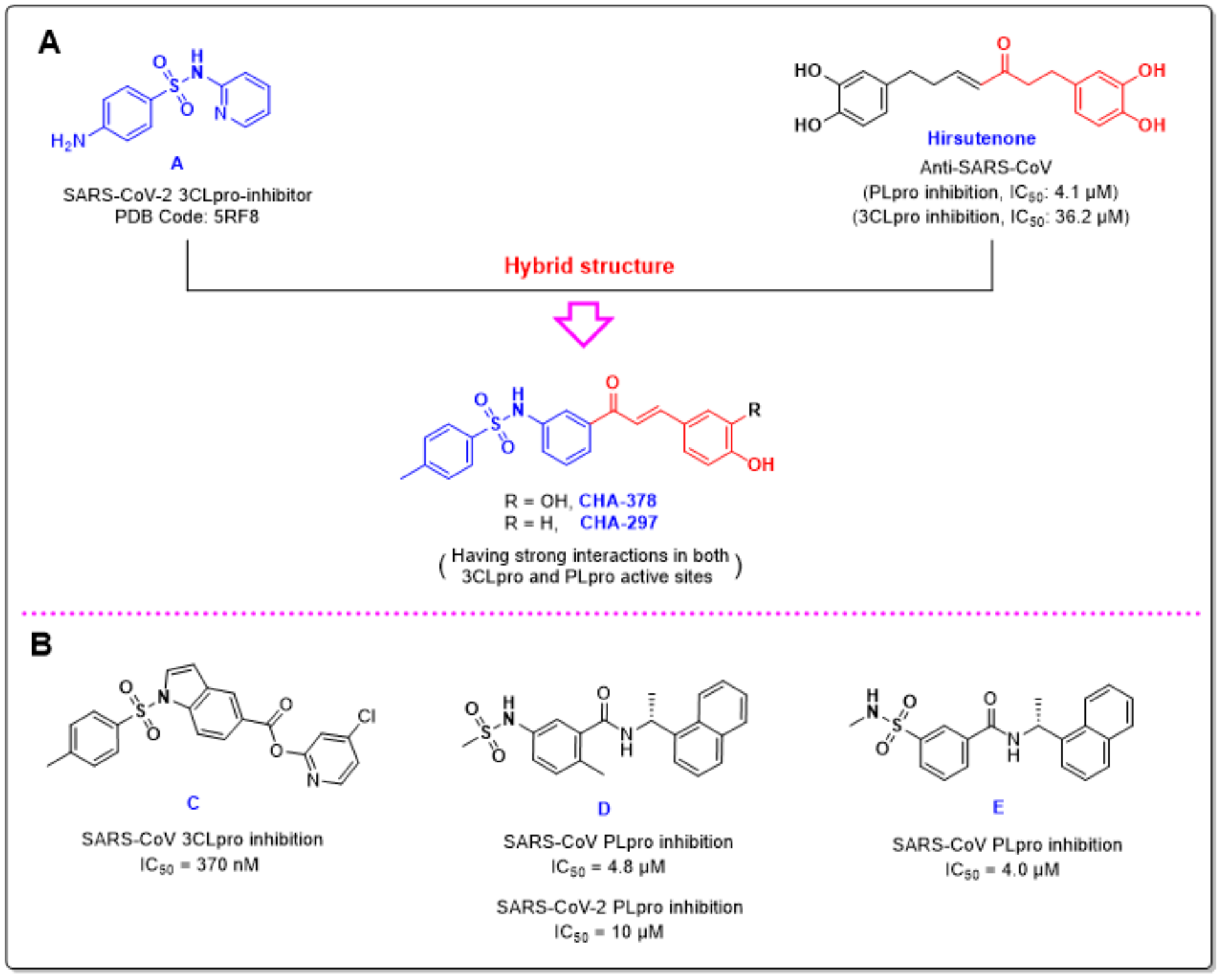

\section{Figure 11}

The chemical structure of CHA-297, CHA-378 and its structural relationship with compound A, hirsutenone, compound C 110, and compounds D and E 111. 

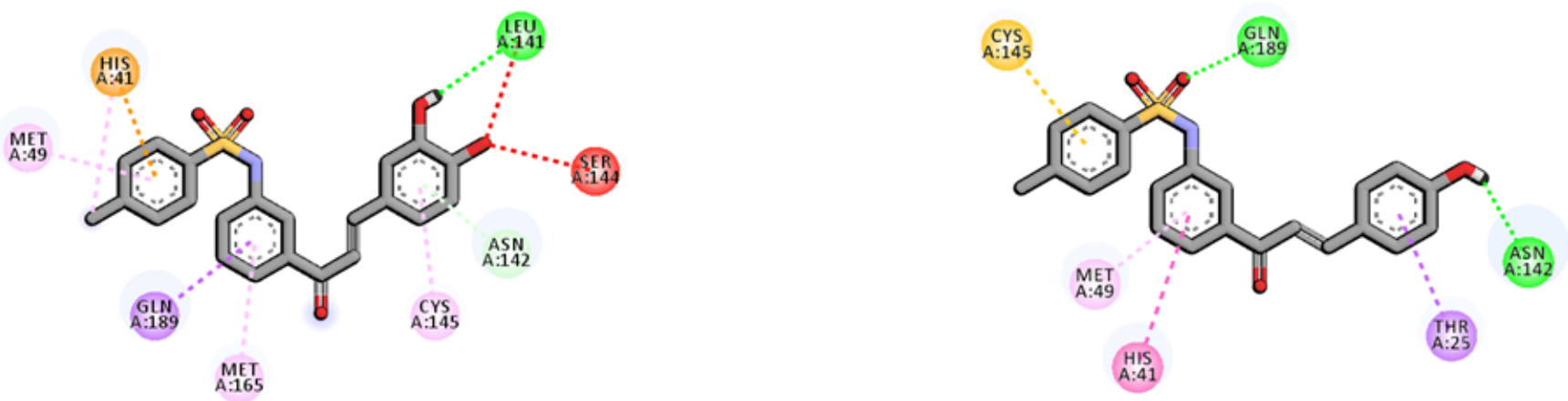

Intractions:

Conventional H-Bond

Pi-Sigma

Unfavorable Acceptor-Acceptor

Pi-Cation

Pi-Pi Stacked

Pi-Alkyl

Intractions:

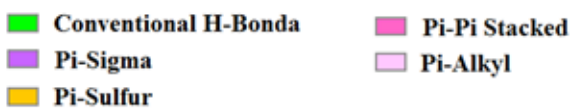

Pi-Donor H-Bond
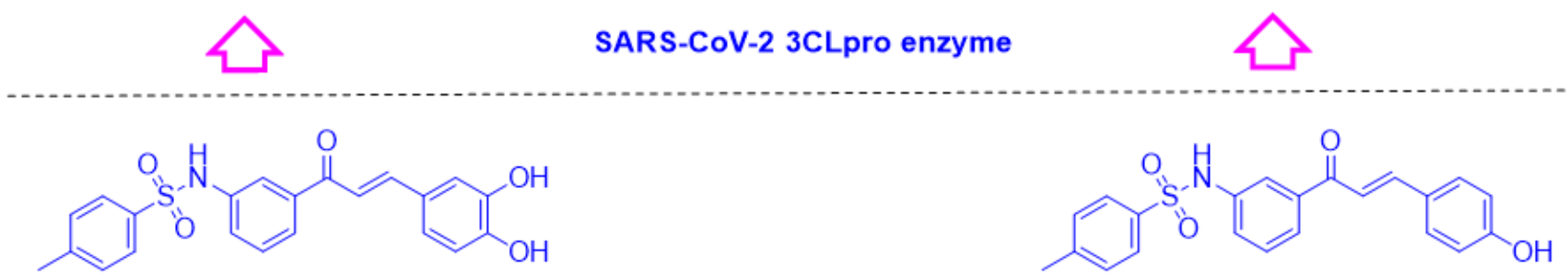

CHA-378

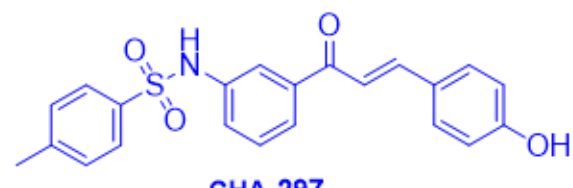

CHA-297

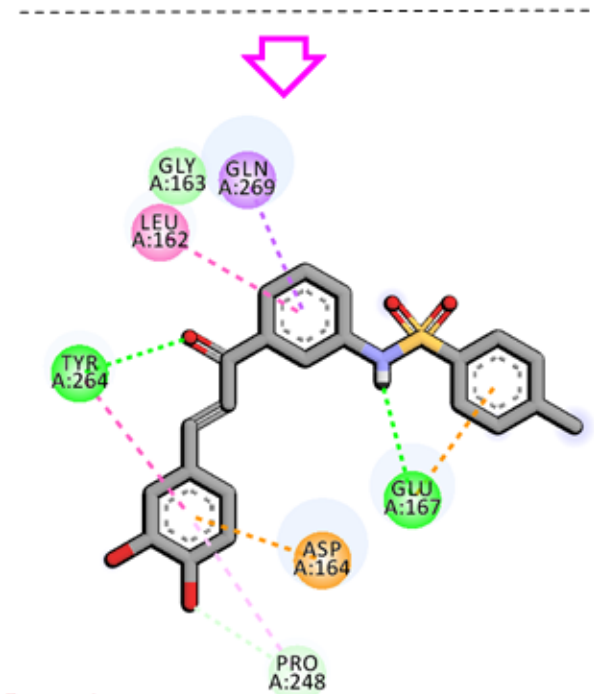

SARS-CoV-2 PLpro enzyme

Intractions:

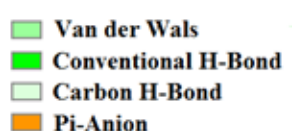

$$
\begin{aligned}
& \text { Pi-Sigma } \\
& \text { Pi-Pi T-Shaped } \\
& \text { Amide-Pi Stacked } \\
& \square \text { Pi-Alkyl }
\end{aligned}
$$

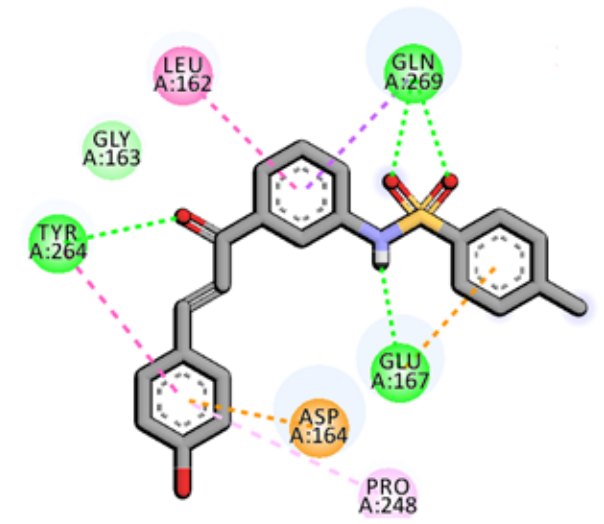

Intractions:

$\begin{array}{ll}\square \text { Van der Waals } & \text { Pi-Pi T-Shaped } \\ \text { Conventional H-Bond } & \\ \text { Pi-Anion } & \square \text { Pid-Alky Stacked } \\ \text { Pi-Sigma } & \end{array}$

\section{Figure 12}

Interactions of CHA-297 and CHA-378 in the active site of the SARS-CoV-2 3CLpro and PLpro enzymes. 




\section{Figure 13}

Structural similarity of the identified selective-3CLpro ligands CHA-233, CHA-236, CHA-383, CHA-384, CHA-392, and CHA-397, and the recently reported biaryl urea compounds $B$ and $E$, and their isosteric amide-analogues compounds $\mathrm{F}$ and $\mathrm{G}$ as special fragments introduced for the design and development of 3CLpro-inhibitors. 


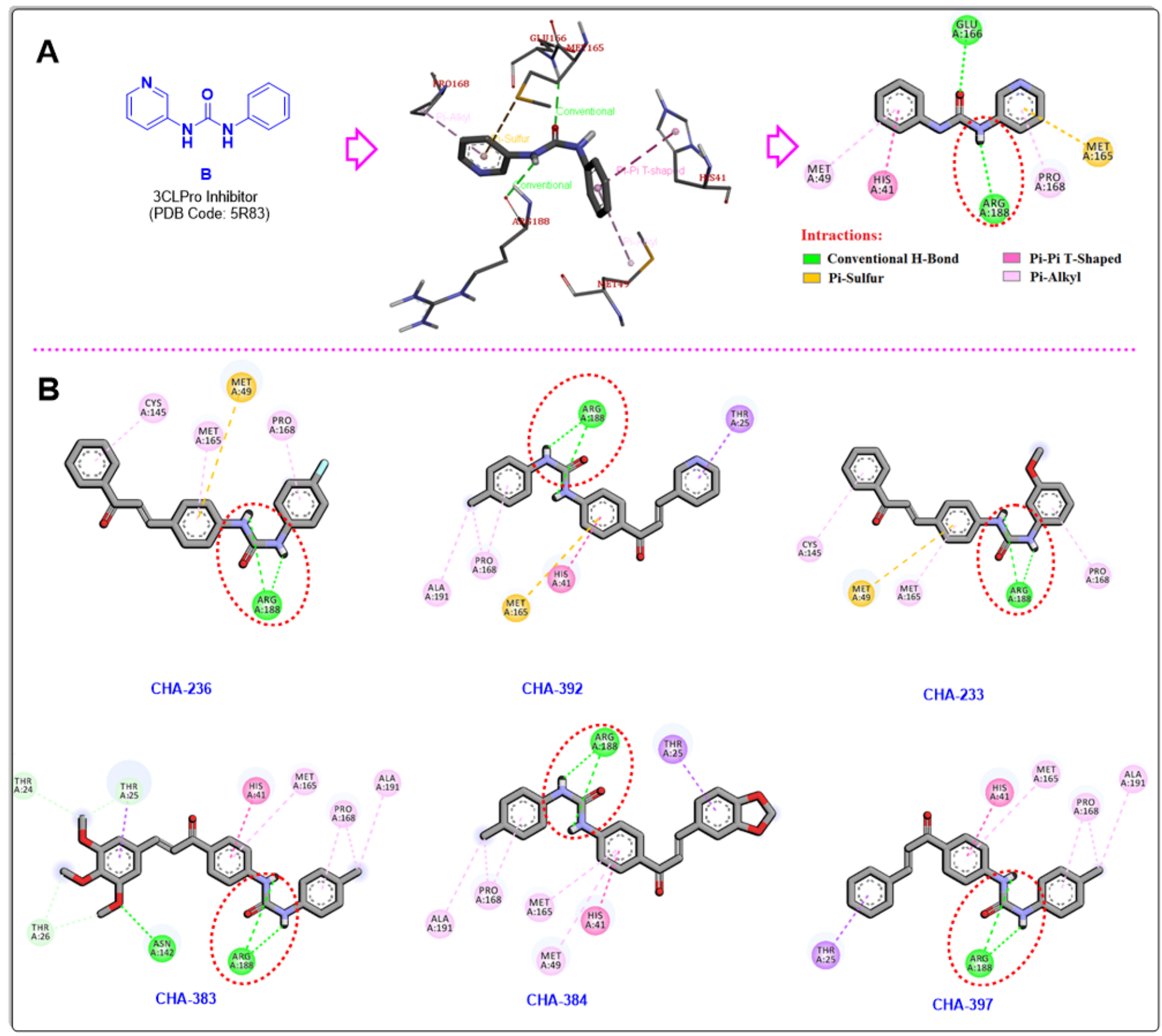

Figure 14

Establishment of the two hydrogen bonds formed in the active site of the SARS-CoV-2 3CLpro by the ureide-chalcone hybrid structures CHA-233, CHA-236, CHA-383, CHA-384, CHA-392, and CHA-397 and carbonyl group of Arg188. 


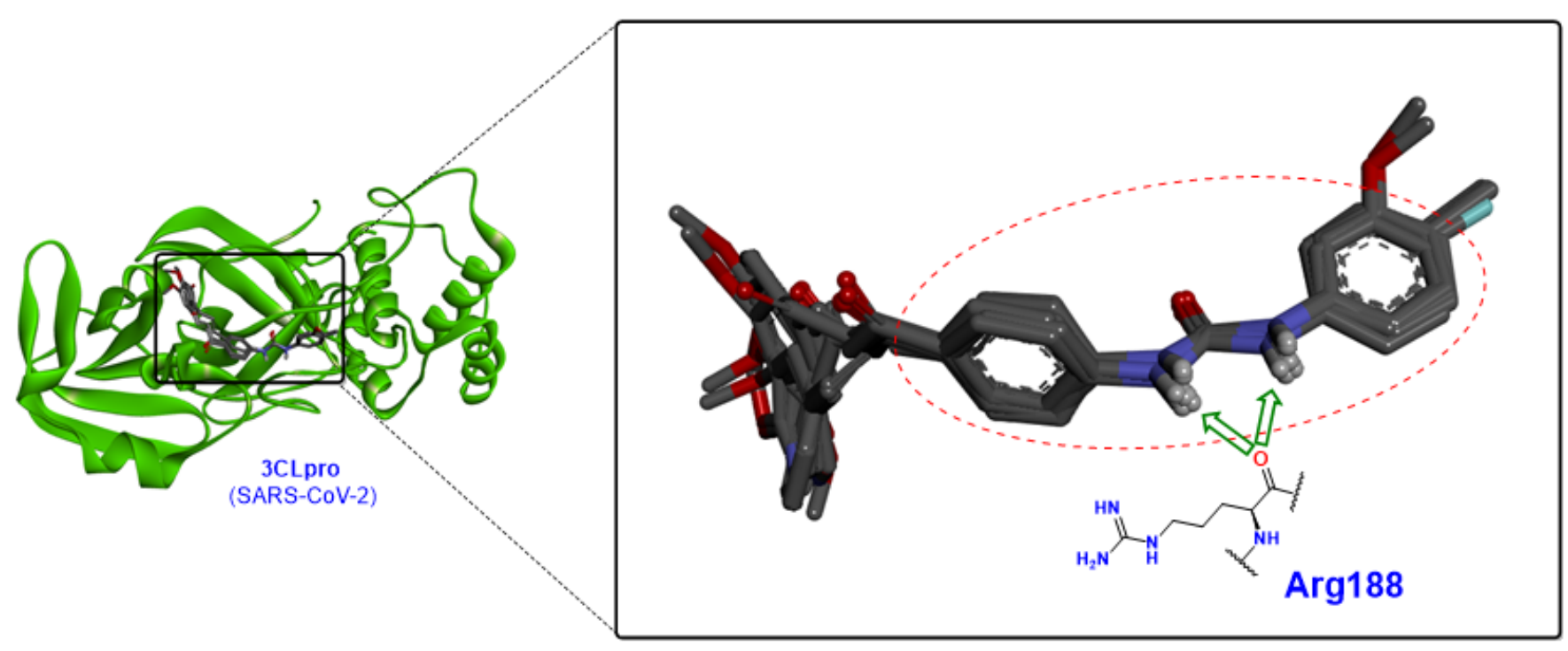

Figure 15

Spatial orientation and establishment of the two hydrogen bonds by the ureide-chalcone hybrid structures CHA-233, CHA-236, CHA-383, CHA-384, CHA-392, and CHA-397 with the carbonyl group of Arg188, at the active site of the SARS-CoV-2 3CLpro. 


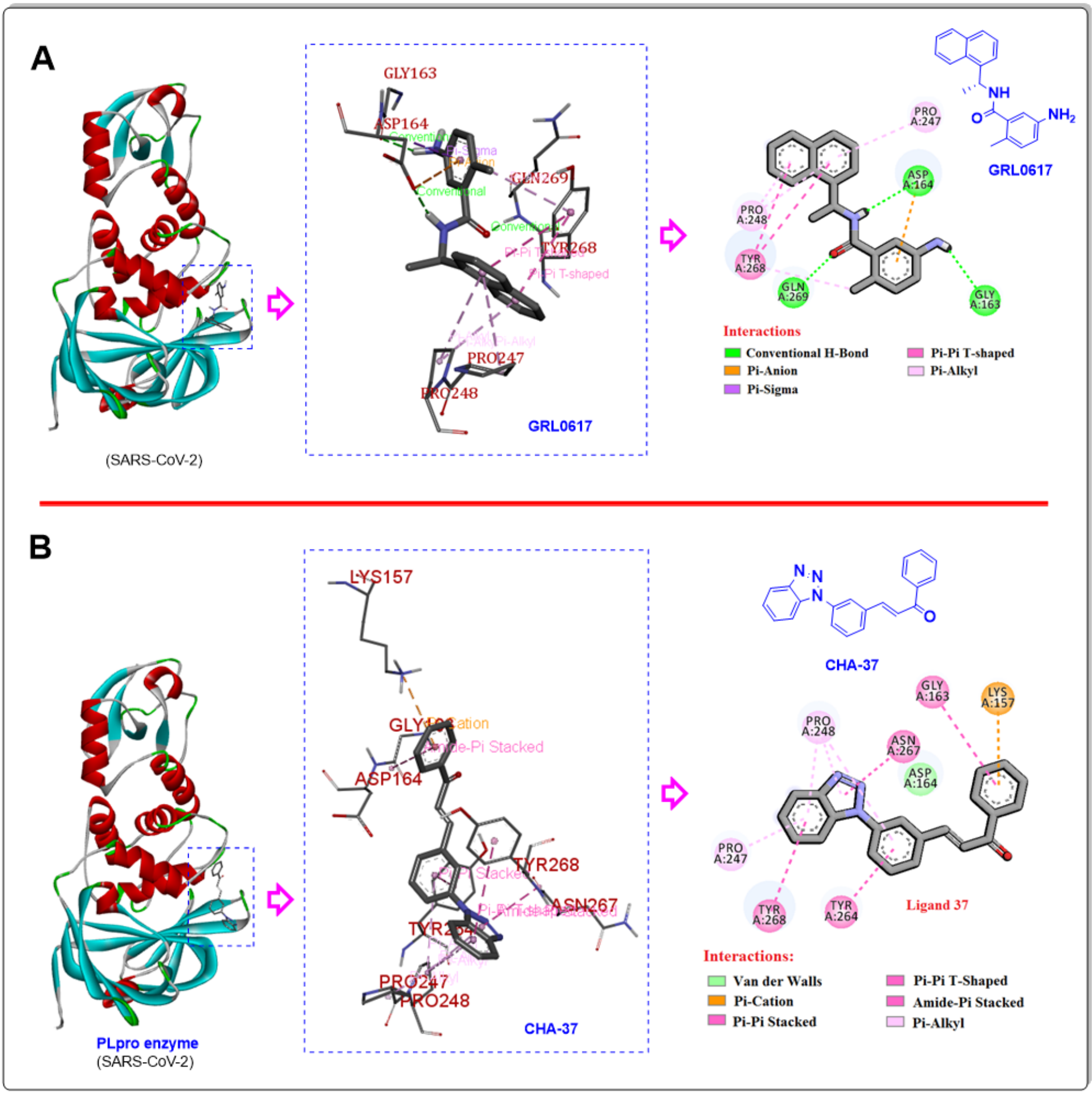

Figure 16

2D and 3D intractions of GRL0617 (as a standard SARS-CoV-2 PLpro inhibitor) and CHA-37 in the active site of the SARS-CoV-2 PLpro enzyme. 


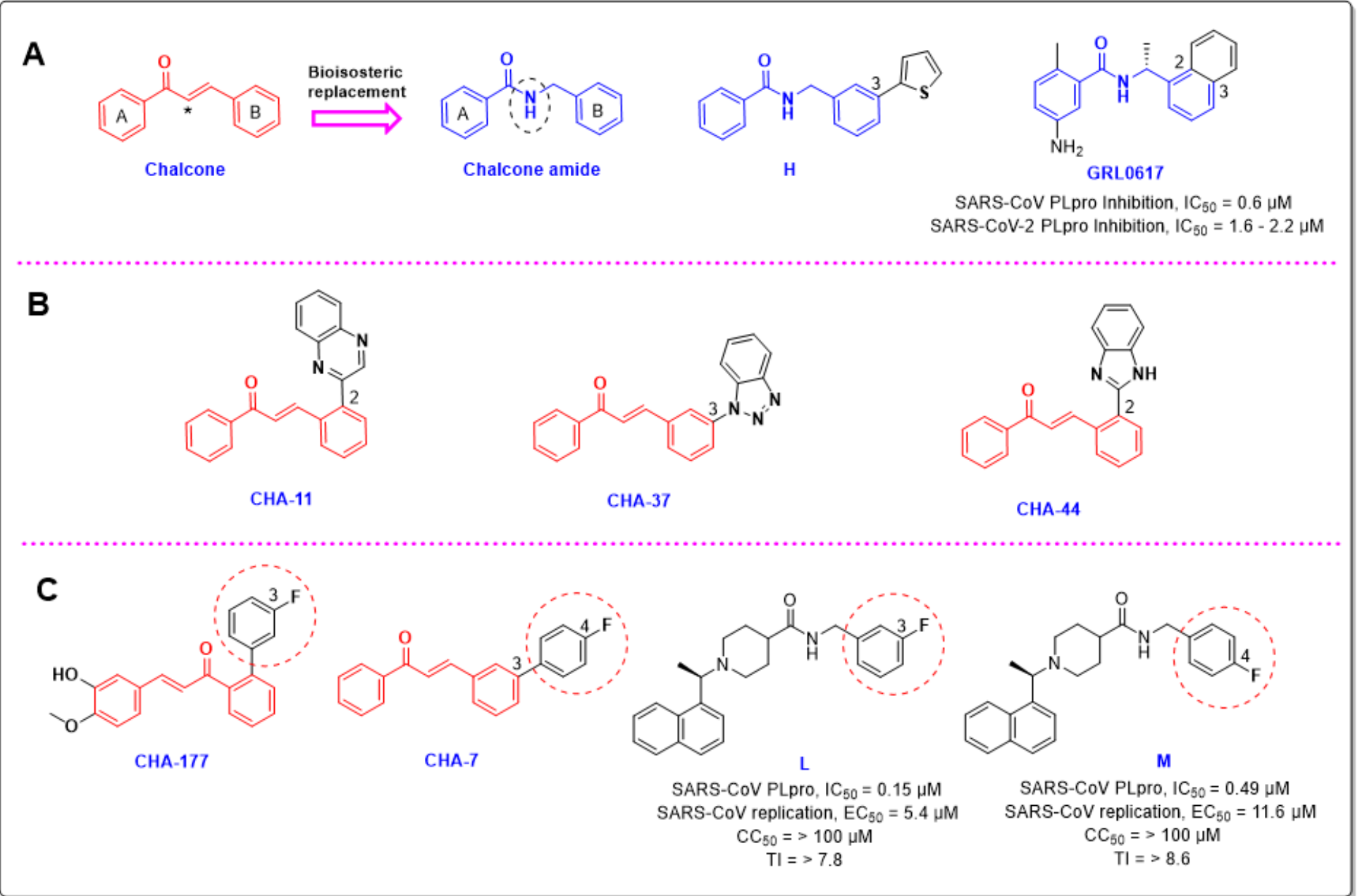

\section{Figure 17}

Structural relationship of the chalcone and the chalcone amide scaffolds, and chemical structure of GRL0617 as a well-known inhibitor of SARS-CoV PLpro, and the predicted selective ligands (CHA-7, CHA11, CHA-37, and CHA-44) toward SARS-CoV-2 PLpro. 


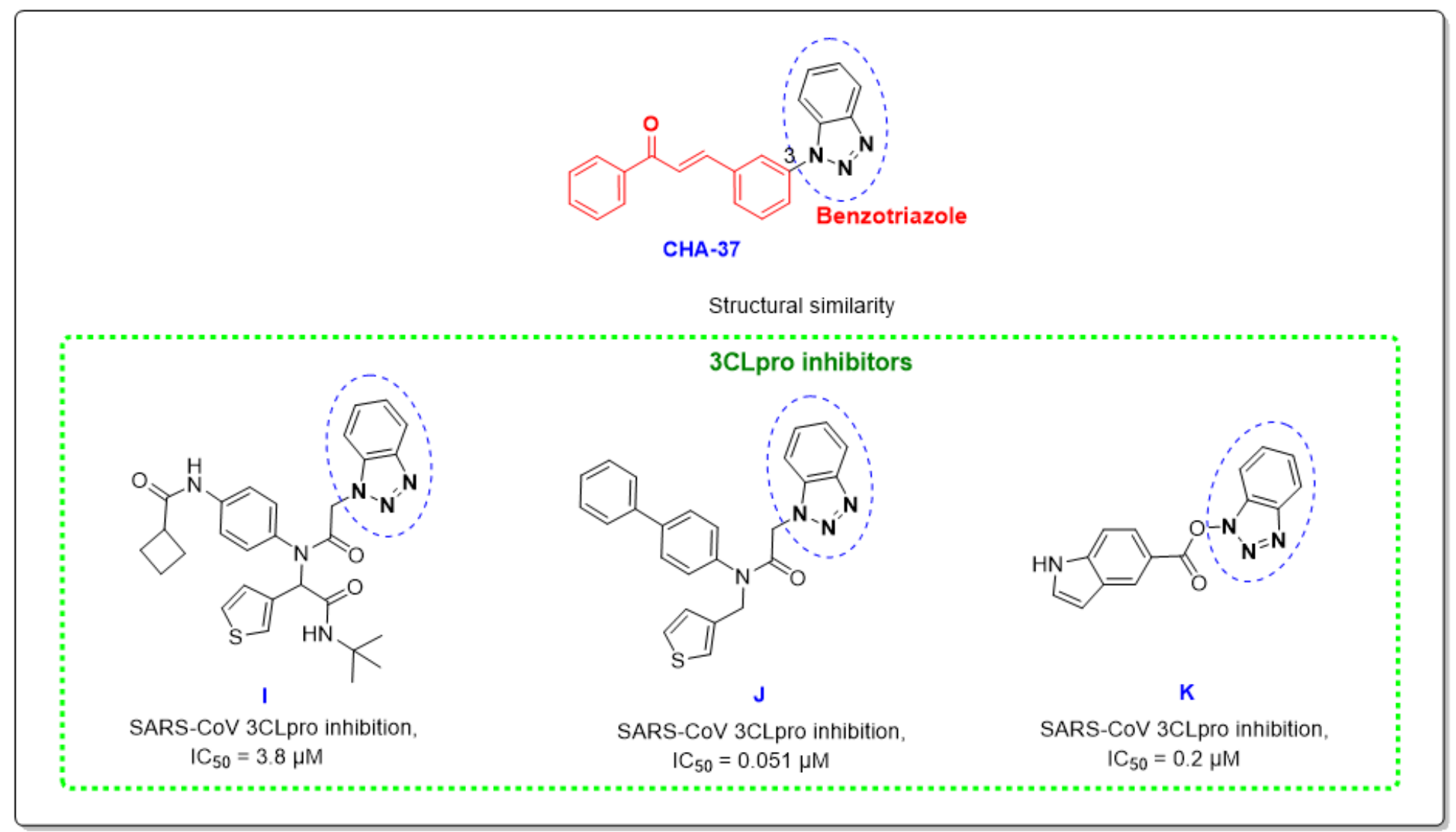

\section{Figure 18}

chemical structures of CHA-37 and some potent SARS-CoV 3CLpro inhibitors containing benzotriazole scaffold. 


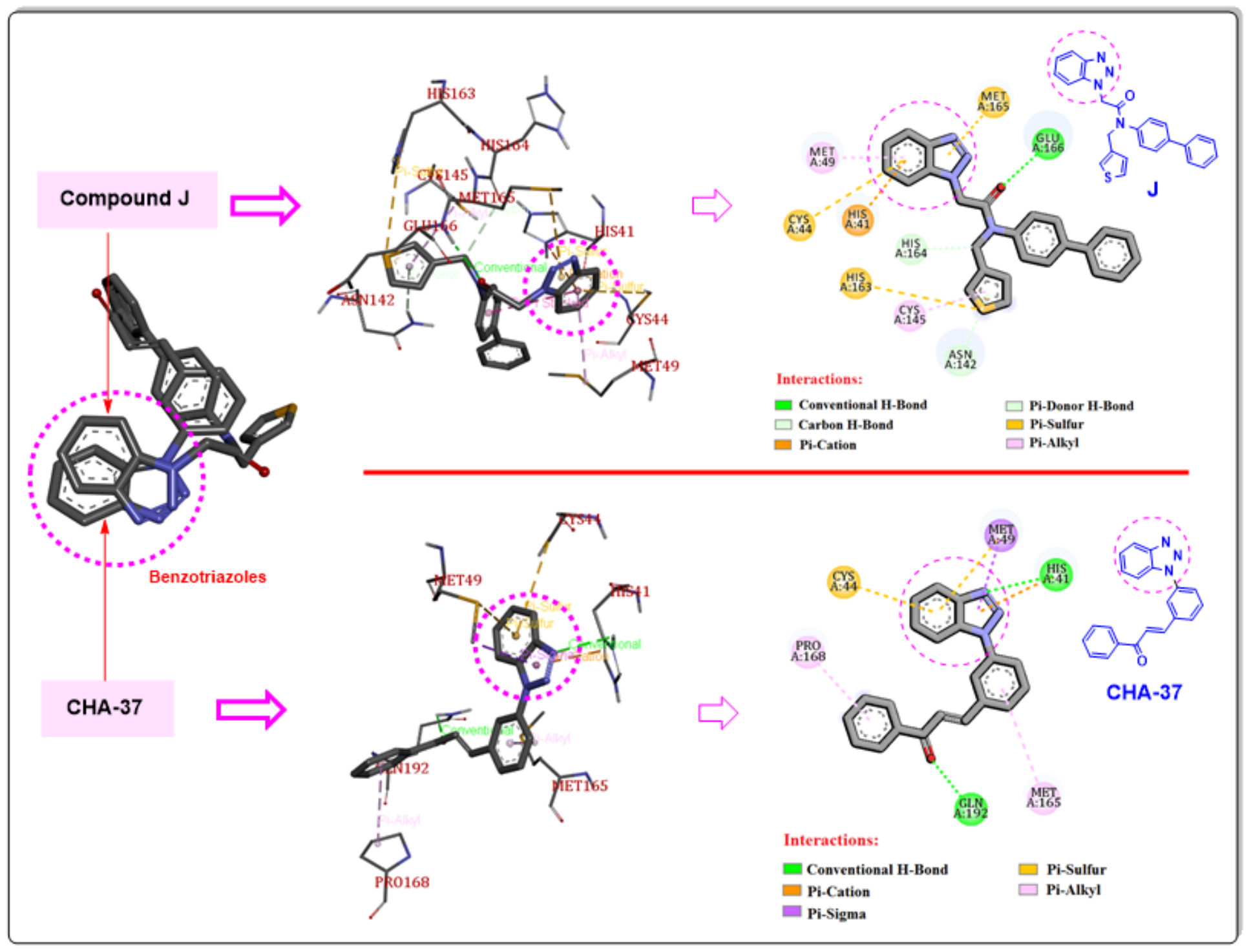

Figure 19

2D and 3D intractions of compound $\mathrm{J}$ as a highly potent SARS-CoV-2 3CLpro inhibitor and CHA-37 in the active site of the 3CLpro enzyme. 